\title{
Fiber orientation in the frontal region of a center-gated disk: Experiments and simulation
}

\author{
S.M. Mazahir ${ }^{1}$, G.M. Vélez-García ${ }^{2}$, *P. Wapperom ${ }^{3}$, and D. Baird ${ }^{l}$ \\ ${ }^{1}$ Department of Chemical Engineering, Virginia Tech, Blacksburg, VA, 24061, USA \\ ${ }^{2}$ Macromolecules and Interfaces Institute, Virginia Tech, Blacksburg, VA, 24061, USA \\ ${ }^{3}$ Department of Mathematics, Virginia Tech, Blacksburg, VA, 24061, USA \\ syedm@vt.edu,gvelez@vt.edu, pwappero@math.vt.edu,dbaird@vt.edu \\ *Author to whom correspondence should be addressed; Peter Wapperom, Tel: +1 540231 7252, Fax: +1
}

5402315960

\begin{abstract}
Fiber orientation in the frontal region of a center-gated disk was measured for the first time and compared with orientation predictions using standard Folgar-Tucker and the reduced strain closure (RSC) model in coupled flow simulations. Fiber orientation was experimentally measured along three different heights representative of shell, transition and core layers, in order to understand the evolution of orientation along the radial direction in the frontal region. Orientation predictions of the Folgar-Tucker model and its two modified versions, the delayed Folgar-Tucker model and the RSC model were assessed against the measured experimental data. Orientation predictions with all three models showed a drop in orientation near the front, which was in qualitative agreement with the experimental data. Modified versions of the Folgar-Tucker model showed a relatively larger drop in orientation in the shell layer with predictions being relatively closer to experimental values. However, no significant slowdown was observed with the modified versions in the transition and core layers. With coupling of flow and orientation, the frontal flow region was slightly larger and orientation predictions showed only slight improvement. A significant improvement in the frontal region was obtained when a lower value of the interaction coefficient was used.
\end{abstract}

\section{INTRODUCTION}

Short glass fiber thermoplastic composites made by injection molding are finding increased usage as high-strength light-weight materials in various applications. The mechanical, electrical, and thermal properties of these materials are significantly affected by the orientation distribution of reinforcing fibers 
which develops during the molding operation. Properties of the final solidified part vary throughout the part due to local variations in the orientation of fibers. This is because of the presence of a complex flow field inside the mold which induces orientation of fibers, a phenomenon known as flow-induced orientation. Therefore, in order to precisely control fiber orientation in a molded composite part, it becomes necessary to develop theoretical models and numerical schemes that can accurately predict fiber orientation under complex flow fields.

Fountain flow [1], is a characteristic flow feature associated with the advancing front in injection molding operations that plays a prominent role in the orientation of fibers in injection molded geometries $[2,3]$. Previous experimental studies on fiber orientation in injection molded geometries such as a centergated disk have shown that the orientation along the flow direction is not the highest at the mold walls but at locations away from the mold walls [4-6]. From these studies, the drop in orientation near the mold walls may be attributed to fountain flow effects, thermal effects or a combination of the two. However, these studies only report experimentally measured fiber orientation data upto a significant distance behind the front and exclude the frontal region of flow. Mazahir et al. [4] have reported fiber orientation measurements along the direction of flow at three different heights representative of the shell, transition and core layers. However, the measured region covers the flow length of the disk only upto $7 \mathrm{r} / \mathrm{H}$ behind the front. Other publications have reported fiber orientation data measured along the thickness of the disk at sparse radial locations [5, 6]. Bay and Tucker [5] conducted the first experimental study on fiber orientation in a center-gated disk and reported $z / H$ profiles only upto a distance $7.5 \mathrm{r} / \mathrm{H}$ behind the front. Vélez-García et al. [6] reported $z / H$ profiles of orientation only upto a distance $7 r / H$ behind the front. In order to gain insights into the effects of the fountain flow on the orientation of fibers in the frontal region of an injection molded geometry such as a center-gated disk, it is crucial to characterize the fiber orientation in the frontal region of the disk.

The Folgar-Tucker (F-T) model [7] has been the most popular model for fiber orientation predictions in injection molded geometries, with varying degrees of success [8-16]. Folgar and Tucker [7] introduced an isotropic rotary diffusion term in the Jeffery's model [17] to account for interaction between 
neighboring fibers in a fiber suspension. However, in fiber suspensions of commercial interest, rheological studies have shown that the evolution of fiber orientation in startup of shear is much slower than what the F-T model predicts $[18,19]$. It has been suggested that reduction in the rate of rotation of fibers is caused by interactions between fibers, at high fiber concentrations [20, 21]. To slow down orientation kinetics in concentrated fiber suspensions, two modifications to the Folgar-Tucker model have been proposed [19, 21, 22]. One of the proposed modifications is the addition of a delay parameter known as the slip parameter $[18,19]$ or the strain reduction factor (SRF) [22], which renders the orientation model non-objective. The other modification is the reduced strain closure (RSC) model which achieves the slowdown in orientation evolution while retaining the objectivity of the original model [21]. The modified versions of the Folgar-Tucker model result in improved orientation predictions by slowing down the evolution of orientation in shear flows such as start-up of shear and shear-flow reversal $[18,19,21]$.

In early simulation studies on fountain flow effects in injection molded geometries, a two-step approach was followed. First the orientation was predicted in the lubrication region using the Hele-Shaw flow approximation and then a separate simulation was performed for the fountain flow region [10, 23]. Such schemes with separate fountain flow simulations made inherent assumptions about the distance behind the front where the fountain flow starts and the velocity profile at the start of the fountain flow region. Chung and Kwon [2], and Park and Kwon [3] used the pseudo-concentration method of Haagh and Van De Vosse [24] to include the effects of fountain flow on fiber orientation in a center-gated disk. Chung and Kwon [2] used the F-T model [7] for orientation predictions in a center-gated disk made with nylon $6 / 6$ and $43 \mathrm{wt} \%$ (fiber volume fraction $\square=0.23$ ) short glass fibers. However, the parameter $C_{I}$ that represents inter-fiber interactions in the F-T model was selected as $C_{I}=0.001$, which is considerably lower than the typical range for short glass fibers $(0.006-0.01)$, recently proposed by Phelps and Tucker [25]. Indeed, values of $C_{I}$ determined from rheological fitting $[4,6,21]$ for the $30 \mathrm{wt} \%$ fiber suspension considered in this paper are an order of magnitude higher than the value used by Chung and Kwon [2]. Park and Kwon [3] used the RSC model for orientation predictions in a center-gated disk and a phenomenological model for $C_{I}$ that includes nonlinear viscoelasticity of the polymer and kinematic 
interactions between fibers and the polymer. Moreover, in these two studies, orientation predictions have been compared with the experimental data in the thickness direction only upto a distance $7.5 \mathrm{r} / \mathrm{H}$ behind the front $[2,3]$. Therefore, with these experimental comparisons and parameter selection, it is difficult to quantify the improvements gained in orientation predictions in the frontal region of a center-gated disk.

This paper has two primary objectives. The first objective is to report the measured fiber orientation data in the frontal region of a thin center-gated disk along the radial direction at three different heights representative of the shell, transition, and core layers. The second objective is to assess predictions of the F-T model and its two modified versions in the frontal region of a center-gated disk in fountain flow simulation.

\section{PROBLEM DESCRIPTION}

A center-gated disk was injection molded using a $30 \mathrm{wt} \%$ (volume fraction $\square=0.1766$ ) short glass fiber polybutylene-terephthalate (PBT) suspension (Valox 420). The viscosity of the PBT matrix was measured on a Rheometrics Mechanical Spectrometer (RMS-800) from steady shear and dynamic oscillatory measurements conducted at $533 \mathrm{~K}$. The viscosity of the matrix showed a Newtonian behavior between shear rates $0.1 \mathrm{~s}^{-1}$ and $100 \mathrm{~s}^{-1}$ with $\not=350 \mathrm{~Pa} \cdot \mathrm{s}$ [20]. The number average fiber diameter $d=12.9$ $\mu \mathrm{m}$ and fiber length $l=364 \mu \mathrm{m}$ were determined from a burn-off process for about 1000 fibers. The measured number average fiber diameter and length correspond to $l / d=28.2$. Weight average fiber length was calculated to be $l_{w}=439 \mu \mathrm{m}[19]$.

A short-shot disk was made with average measurements, inner radius $R_{i n}=2.97 \mathrm{~mm}$, outer radius $R_{\text {out }}=51.8 \mathrm{~mm}$ and thickness $2 H=1.38 \mathrm{~mm}$ as shown in Fig. 1(a). In order to exclude the effects of packing, the radial distance filled inside the mold was about $90 \%$ of the total radial length of the mold. The resin was first dried overnight in a vacuum oven at a pressure less than $1.35 \mathrm{kPa}$ and the dried resin was fed to the screw under nitrogen atmosphere at $433 \mathrm{~K}$. The suspension was injected into the mold maintained at $363 \mathrm{~K}$ using an injection pressure of approximately $20 \mathrm{MPa}$, with filling time 
approximately $1 \mathrm{sec}$. This corresponds to a volumetric flow rate $q \approx 11.8 \mathrm{~cm}^{3} / \mathrm{s}$. The disk was allowed to cool and solidify for about 1 min inside the mold to minimize warpage.

\section{EXPERIMENTAL EVALUATION OF THE FRONTAL REGION}

\subsection{Shape and texture of the advancing front}

The shape and the texture of the advancing fronts of $30 \mathrm{wt} \%$ short glass fiber / PBT suspension and pure PBT polymer were analyzed under an optical microscope. The center-gated disk of pure PBT was injection-molded under the same conditions of temperature and pressure as the disk made of the fiber suspension. The frontal region of each disk was cut out and mounted in acrylic and observed in the $r z-$ plane under an optical microscope at 5X zoom level. Fig. 2 shows the images of the advancing front of the two disks injection molded with fiber suspension and pure polymer, respectively. As can be seen from the figures, the shapes of the two fronts are very different. The front of the suspension shows an almost semi-circular shape and the contact point with the mold wall is at an approximate radial distance of 1.3 $r / H$ from the tip of the front. The front of the fiber suspension has a rough texture and voids can be seen in the frontal region. The front of pure polymer shows a protrusion coming out from the center of the front while the contact point with the mold wall seems to be farther behind compared to the contact point in case of fiber suspension. The front of pure polymer shows a smooth texture and no significant voids or air bubbles can be seen in the frontal region.

\subsection{Experimental orientation in the frontal region}

Measurements of fiber orientation in the fountain flow region were made at constant thickness-wise positions along the radial direction in the frontal region of the center-gated disk. The $z / H$ heights were selected as $z_{s} / H=0.75,0.42$, and 0.08 , which are representative of the shell, transition and core layers, respectively. The measurements were made in small rectangular bins with height $H / 6$ and a width of twice the fiber length $2 l$, respectively, as described by Vélez-García et al. [26]. The sampling bins were centered in the $z$-direction at selected $z_{s} / H$ heights. As shown in Fig. 3, six sampling locations were selected in each layer to characterize the evolution of orientation. The positions of these locations along the radial 
direction are measured from the tip of the advancing front. The advancing front (melt-air interface) in this reference frame corresponds to a radial position $r / H=0$. The first five locations for sampling were centered in the $r$-direction at $r_{s} / H=-7.0,-5.6,-4.9,-3.5$ and -2.1 from the front. These correspond to flow lengths of $90 \%, 92 \%, 93 \%, 95 \%$ and $97 \%$ respectively. For the sixth location, we considered a position $r_{s} / H=-0.7$ (corresponding to $99 \%$ of the flow length) in the core and transition layers and $r_{s} / H=-1.4$ (corresponding to $98 \%$ of the flow length) in the shell layer. The last location in the shell layer was taken at $98 \%$ because at $99 \%$, the sampling bin crossed over the polymer-air interface and the polymer phase covered only a part of the bin. In this way, we could ensure a sufficient number of fibers in every bin, to obtain reliable orientation data [26]. The average number of fibers in a bin is about 128.

Therefore, the location at $99 \%$ in the shell layer would not give reliable orientation information due to very limited number of fibers available for measurement.

The measurement procedure proposed by Vélez-García et al. [26] was used in the characterization of fiber orientation. This is a modified version of the method of ellipses (MoE) [27]. The MoE considers the elliptical footprints of fibers in the polished plane to determine the orientation angles (i.e. in-plane $\left(\square_{f}\right)$ and out-of-plane $\left(\theta_{f}\right)$ angles) for each individual fiber and estimates the volumetric average of orientation represented through a second-order tensor. Vélez-García et al. [26] modified the MoE to add partially elliptical and rectangular footprints so that reliable orientation data can be obtained from small sampling areas. The modified method also includes a shadow detection that distinguishes between fibers having the same footprint in the polished plane but oriented at angles $\square_{f}$ and $\square_{f}+\pi$ This correction is achieved by identifying a shadow along the major axis of the elliptical or partially elliptical footprint. Orientation measurements were made by an optical microscope at $20 \mathrm{X}$ magnification on images taken at various radial locations, which were $2 l$ wide and contained the sampling area of interest. Using an in-house MATLAB program, the wall-to-wall thickness of the disk in each image was divided into 12 equal bins along the $z$-direction and orientation was determined from elliptical, partially elliptical and rectangular footprints in the selected bins. 
The measured orientation data is shown in Figs. 4 through 6 for the shell, transition, and core layer, respectively. The figures show the diagonal and the off-diagonal components of the orientation tensor at the sampled locations. Fig. 4a shows the diagonal components of the orientation tensor at selected radial locations for the shell layer. Here, $A_{r r}$ values show a steady decrease between radial locations $r_{s} / H=-5.6$ and $r_{s} / H=-1.4$. The changes in $A_{r r}$ are complemented by opposite changes in $A_{\theta \theta}$ in the entire region. The primary direction of orientation changes from the $r$-direction to the $\theta$-direction in the region between $r_{s} / H$ $=-5.6$ and $r_{s} / H=-1.4 . A_{z z}$ remains close to zero at all sampled locations which means that the orientation is planar in $r \theta$-plane in the region from the front to a distance upto $r_{s} / H=-7.0$ behind the front.

The $A_{r z}, A_{r \theta}$ and $A_{\theta z}$ components of the orientation tensor in the shell layer behind the front are shown in Fig. 4b. $A_{r z}$ stays between -0.1 and 0.1 at all sampled locations in the region between $r_{s} / H=-7.0$ and $r_{s} / H=-1.4 . A_{z \theta}$ is almost zero in the entire region between $r_{s} / H=-7.0$ and $r_{s} / H=-1.4 . A_{r \theta}$ has a small negative value at $r_{s} / H=-7.0$ which increases to about 0.2 at $r_{s} / H=-5.6$ and after that levels off at around 0.3 .

The diagonal components of the orientation tensor for the transition layer are shown in Fig. 5a. In the transition layer, $A_{r r}$ shows almost a linear drop from $A_{r r}=0.64$ to $A_{r r}=0.09$ between $r_{s} / H=-7.0$ and $r_{s} / H$ $=-0.7$. The increase in $A_{\theta \theta}$ is almost equal to the reduction in $A_{r r}$ between $r_{s} / H=-7.0$ and $r_{s} / H=-2.1$, a similar behavior as in the shell layer. However, very close to the front, between $r_{s} / H=-2.1$ and $r_{s} / H=-$ 0.7, $A_{\theta \theta}$ is almost flat. In this region, the drop in $A_{r r}$ is complemented by a similar increase in $A_{z z}$ which results in a higher value of $A_{z z}$ compared to $A_{r r}$ at $r_{s} / H=-0.7 . A_{z z}$ remains close to zero at all other sampled locations. The increase in $A_{z z}$ in the region between $r_{s} / H=-2.1$ and $r_{s} / H=-0.7$ suggests that the extension in the $z$-direction starts to dominate in this region and causes the fibers to rotate in the $z$-direction. Fig. $5 \mathrm{~b}$ shows the off-diagonal components of orientation in the transition layer. $A_{r z}$ and $A_{z \theta}$ stay close to zero in the region between $r_{s} / H=-7.0$ and $r_{s} / H=-4.9$ and show a slight drop after $r_{s} / H=-4.9 . A_{r \theta}$ increases from values around 0 to about 0.3 in the region between $r_{s} / H=-7.0$ and $r_{s} / H=-4.9$, stays relatively flat upto $r_{s} / H=-2.1$ and then slightly drops between $r_{s} / H=-2.1$ and $r_{s} / H=-0.7$. 
Fig. 6a shows the diagonal components of orientation in the core layer at selected radial locations in the frontal region. In this layer, due to the absence of high shear, extensional flow dominates, which tends to align fibers in the $\theta$-direction. Therefore, $A_{r r}$ is relatively small in this layer as compared to the shell and transition layers at all radial locations between $r_{s} / H=-7.0$ and $r_{s} / H=-0.7 . A_{r r}$ shows a steady decrease along the radial direction, as was also observed in the transition layer. $A_{\theta \theta}$ stays relatively constant at around 0.8 which represents high orientation along the $\theta$-direction in the entire frontal region. In this layer, the mirroring behavior is similar to the transition layer with $A_{r r}$ and $A_{\theta \theta}$ mirroring each other near $r_{s} / H=-7.0$. The $A_{z z}$ profile in the core layer follows a similar pattern as in the transition layer. The off-diagonal components of orientation in the core layer are shown in Fig. 6b. $A_{r z}$ and $A_{z \theta}$ stay close to zero while $A_{r \theta}$ shows a similar profile as in the transition layer.

\section{GOVERNING EQUATIONS}

\subsection{Orientation evolution equations}

For the evolution of orientation of long slender particles such as glass fibers in semi-dilute fiber concentration regimes, Folgar and Tucker [7] proposed a modification to the Jeffery's model [17] by introducing an isotropic rotary diffusion term that accounts for fiber-fiber interaction. The standard Folgar-Tucker model is given by:

$$
\frac{D \mathbf{A}}{D t}=\mathbf{W} \cdot \mathbf{A}-\mathbf{A} \cdot \mathbf{W}+\lambda\left(\mathbf{D} \cdot \mathbf{A}+\mathbf{A} \cdot \mathbf{D}-2 \mathbf{A}_{4}: \mathbf{D}\right)+2 C_{I} \dot{\gamma}(\mathbf{I}-3 \mathbf{A})
$$

where $\mathbf{A}$ is the second-order orientation tensor, $D_{D} / D t$ is the material derivative, $\mathbf{W}=\left[(\nabla \mathbf{v})-(\nabla \mathbf{v})^{T}\right] / 2$ the vorticity tensor, $\mathbf{D}=\left[(\nabla \mathbf{v})+\left(\nabla \mathbf{v}^{T}\right)\right] / 2$ the rate-of-strain tensor, and $\nabla \mathbf{v}=\partial v_{j} / \partial x_{i}$ the velocity gradient. The shape factor $\lambda=\left((l / d)^{2}-1\right) /\left((l / d)^{2}+1\right)$ equals one for long slender particles such as glass fibers. $\mathbf{A}_{4}$ is the fourth-order orientation tensor, I the identity tensor, $C_{I}$ the isotropic interaction coefficient and $\dot{\gamma}=\sqrt{2 \mathbf{D}: \mathbf{D}}$.

The orientation evolution predicted by the Folgar-Tucker model has been tested in transient shear flow and it has been observed that experimentally observed evolution kinetics are much slower than the 
Folgar-Tucker model predicts $[6,21,22]$. Hence, in order to slow down the evolution of orientation predicted by the Folgar-Tucker model, two modifications were proposed recently [19, 21, 22]. First, the introduction of a strain reduction factor (SRF) was proposed by Huynh et al. [22] or a slip parameter $(\alpha)$ proposed by Eberle et al. [19]. We will use the term 'slip parameter' for this factor and 'delayed FolgarTucker model' for the Folgar-Tucker model with this factor. The slip parameter represents the retardation in orientation evolution due to inter-fiber interactions in concentrated suspensions. It is a factor between 0 and 1 and the entire right hand side of the standard Folgar-Tucker model is multiplied with this factor, thereby slowing down the evolution of orientation:

$$
\frac{D \mathbf{A}}{D t}=\alpha\left[\mathbf{W} \cdot \mathbf{A}-\mathbf{A} \cdot \mathbf{W}+\lambda\left(\mathbf{D} \cdot \mathbf{A}+\mathbf{A} \cdot \mathbf{D}-2 \mathbf{A}_{4}: \mathbf{D}\right)+2 C_{I} \dot{\gamma}(\mathbf{I}-3 \mathbf{A})\right]
$$

However, with this modification, the orientation equation becomes non-objective. Wang et al. [21] followed a different approach and proposed a modification to the standard Folgar-Tucker model that achieves the slowdown in evolution of orientation while retaining the objectivity of the model. The modified model is known as the reduced strain closure (RSC) model and consists of a modification in the closure approximation for $\mathbf{A}_{4}$ eliminating the problem of non-objectivity. The RSC model is given by:

$$
\frac{D \mathbf{A}}{D t}=\mathbf{W} \cdot \mathbf{A}-\mathbf{A} \cdot \mathbf{W}+\lambda\left(\mathbf{D} \cdot \mathbf{A}+\mathbf{A} \cdot \mathbf{D}-2\left[\mathbf{A}_{4}+(1-\kappa)\left(\mathbf{L}_{4}-\mathbf{M}_{4}: \mathbf{A}_{4}\right)\right]: \mathbf{D}\right)+2 \kappa C_{I} \dot{\gamma}(\mathbf{I}-3 \mathbf{A})
$$

The strain reduction factor $\kappa$ has a value between 0 and 1 and plays a similar role as the slip parameter $\alpha$. Two additional fourth-order tensors $\mathbf{L}_{4}$ and $\mathbf{M}_{4}$ are introduced in the RSC model which are defined as:

$$
\mathbf{L}_{4}=\sum_{i=1}^{3} \lambda_{i} \mathbf{e}_{i} \mathbf{e}_{i} \mathbf{e}_{i} \mathbf{e}_{i}, \quad \mathbf{M}_{4}=\sum_{i=1}^{3} \mathbf{e}_{i} \mathbf{e}_{i} \mathbf{e}_{i} \mathbf{e}_{i}
$$

where $\lambda_{i}$ are the eigenvalues and $\mathbf{e}_{i}$ the eigenvectors of $\mathbf{A}$.

A closure approximation needs to be used to express $\mathbf{A}_{4}$ in terms of $\mathbf{A}$. The invariant-based optimal fitting (IBOF) closure approximation was proposed by Chung and Kwon [28] to express $\mathbf{A}_{4}$ in terms of $\mathbf{A}$. The accuracy of the IBOF closure is as good as eigenvalue-based closures, but required computational time is less. We use the IBOF closure approximation for $\mathbf{A}_{4}$ in all simulations. 


\subsection{Equations of motion}

The mass and momentum equations for the flow of an incompressible fluid under creeping flow conditions are:

$$
\begin{gathered}
\nabla \cdot \mathbf{v}=0 \\
\nabla \cdot\left(-p \mathbf{I}+2 \eta_{s} \mathbf{D}+\mathbf{T}_{\text {fiber }}\right)=\mathbf{0}
\end{gathered}
$$

where $\nabla$ is the gradient operator, $\mathbf{v}$ the velocity, $p$ the pressure, $\mathbf{I}$ the identity tensor, and $\eta_{s}$ the matrix viscosity. $\mathbf{T}_{\text {fiber }}$ is the viscous extra-stress contribution from the fibers to the total stress in the fluid, given by

$$
\mathbf{T}_{\text {fiber }}=2 v \zeta_{s t r} \mathbf{D}: \mathbf{A}_{4}
$$

where $v$ is the concentration of the fibers and $\zeta_{t r}$ is the viscous drag coefficient between the fibers and the polymer matrix [29]. We refer to $\zeta_{t r}$ as the coupling parameter. The general form of $\mathbf{T}_{\text {fiber }}$ contains more terms, but most of those terms are negligible for our fibers. Dinh and Armstrong [29] proposed a model for the viscous drag coefficient $\zeta_{t r}$ in semi-concentrated fiber suspensions: 


$$
\zeta_{t r}=\frac{\pi \eta_{s} l^{3}}{12 \ln \left(2 a_{c} / d\right)}
$$

where $a_{c}$ is the average inter-fiber distance that depends on the fiber orientation. For $\mathbf{A}_{4}$ appearing in the extra-stress term, we use the IBOF closure approximation in all simulations.

Isothermal filling conditions are assumed because of fast filling conditions, (about one second filling time), in agreement with the findings of Wu et al. [30]. The amount of heat dissipated through viscous heating was approximately equal to the heat loss due to conduction through the mold walls. The maximum temperature rise due to viscous dissipation was estimated to be about $3.5 \mathrm{~K}$.

The flow equations are solved in a non-dimensional framework. Velocities are scaled with the average radial velocity $v_{\text {avg }}\left(R_{\text {in }}\right)$ at the inlet of the mold, lengths are scaled with the half-thickness of the disk $H$, and time with $v_{\text {avg }}\left(R_{\text {in }}\right) / H$. The coupling parameter $k_{s t r}^{c}$ is scaled with the matrix viscosity $\eta_{s}$.

\subsection{Model parameters}

The model parameters contained in the orientation evolution equations, Eqs. (1-3), have been previously determined for Valox 420 from experimental orientation data in startup of shear flow [4]. The 
parameter values are $C_{I}=0.0112$ for the standard Folgar-Tucker model, $\left(C_{I}, \alpha\right)=(0.0112,0.40)$ for the delayed Folgar-Tucker model, and $\left(C_{I}, \kappa\right)=(0.0112,0.40)$ for the RSC model. The value of the coupling parameter $\xi_{s t}$, is in general, obtained either from theory or from rheological experiments of fiber suspensions. Mazahir et al. [4] fitted a non-dimensional value of $\boldsymbol{\zeta}_{t r}^{\zeta}=6.18$ to steady state stress data in startup of simple shear flow obtained by Eberle et al. [20]. For our fiber suspension, with $l / d=28$ and = 0.1766, the theoretical value of $\zeta_{t r}$ is 37 based on Dinh and Armstrong theory [29].

\section{NUMERICAL METHODS}

Filling of a center-gated disk mold with a short glass fiber suspension is simulated by solving a transport equation for the evolution of the advancing front, equations of motion for the velocity and pressure, and orientation equations for the fiber orientation. In the time-stepping scheme, these three equations are solved in a decoupled fashion at every time step. For the spatial discretization of velocity, continuous biquadratic polynomials are used, while pressure is represented by continuous bilinear polynomials. For the orientation and extra-stress, we use discontinuous biquadratic and discontinuous bilinear polynomial representation, respectively. The pseudo-concentration variable in the transport equation is discretized in space with discontinuous biquadratic polynomials. The choice of discontinuous polynomials for the orientation, extra-stress and pseudo-concentration is due to the hyperbolic nature of the orientation and transport equations. The details of solution schemes for these equations are given below. Equations of motion are discussed first, followed by the orientation and transport equations.

\subsection{Equations of motion}

The equations of motion (5), (6) are solved simultaneously for the polymer and air phase at every time step. The velocity $\mathbf{v}^{n+1}$ and pressure $p^{n+1}$ at time level $t^{n+1}$ are obtained from simultaneous solution of

$$
\begin{gathered}
\nabla \cdot \mathbf{v}^{n+1}=0 \\
\nabla \cdot\left(-p^{n+1} \mathbf{I}+2 \eta_{s} \mathbf{D}^{n+1}+\tilde{\mathbf{T}}_{\text {fiber }}^{n+1}\right)=\mathbf{0}
\end{gathered}
$$


Viscosity $\eta_{s}$ is taken to be polymer viscosity in the polymer phase and air viscosity in the air phase. This is achieved throug the use of the pseudo-concentration variable $c$ as

$$
\eta_{s}=c \times \eta_{p}+(1-c) \times \eta_{a}
$$

where $\eta_{p}$ is the polymer viscosity and $\eta_{a}$ the air viscosity. The pseudo-concentration variable lies in the range $[0,1]$. A value of $c=1$ indicates the polymer phase, a value of $c=0$ air phase and a value $c=0.5$ defines the polymer-air interface. Near the polymer-air interface, $\eta_{s}$ is linearly interpolated between the viscosity values for the polymer and air phase. Air is considered to have zero density and viscosity about $0.1 \%$ of the fiber suspension. With this selection of material properties for air, the contribution of air to pressure buildup in the mold is negligible as compared to that from the fiber suspension. For coupled simulations, the viscous extra-stress contribution from fibers is considered through the term $\tilde{\mathbf{T}}_{\text {fiber }}^{n+1}=2 c^{n+1} v \zeta_{\text {str }} \mathbf{D}^{n+1}: \mathbf{A}_{4}^{n}$, where $\mathbf{D}$ is implicit at time level $t^{n+1}$ and $\mathbf{A}_{4}$ is explicit at time level $t^{n}$. The spatial discretization of equations of motion is performed with the Galerkin finite element method. The value of $c$ is determined at every integration point, $c=0$ is used if $c<0$, and $c=1$ is used if $c>1$ in order to eliminate non-physical values of $c$ due to non-linear-interpolation. This correction is important because it avoids non-physical viscosity values such as $\eta_{s}<0$ and $\eta_{s}>\eta_{p}$.

At the inlet of the mold, a parabolic velocity profile is prescribed. At other boundaries, nondimensional boundary conditions are prescribed as [24]

$$
\begin{array}{ll}
a v_{t}+\sigma_{t}=0 & \forall x \in \Gamma_{\mathrm{w}} \cup \Gamma_{\mathrm{o}} \\
v_{n}=0 & \forall x \in \Gamma_{\mathrm{w}} \\
a v_{n}+\sigma_{n}=0 & \forall x \in \Gamma_{\mathrm{o}}
\end{array}
$$

where $v_{t}$ and $v_{n}$ are tangential and normal components of the velocity vector $\mathbf{v}$ and $\sigma_{t}$ and $\sigma_{n}$ are tangential and normal components of the stress tensor $\boldsymbol{\sigma}=-p \mathbf{I}+2 \eta_{s} \mathbf{D}+\widetilde{\mathbf{T}}_{\text {fiber }}$. The boundary along the mold walls $\Gamma_{\mathrm{w}}$ and the outlet boundary for air $\Gamma_{\mathrm{o}}$ are shown in Fig. 1(b). The dimensionless parameter $a=$ $10^{10}$ for $c \geq 0.5$ and $a=0$ for $c<0.5$. With the first boundary condition, air is allowed to slip freely along the mold walls while the slip velocity for the polymer along the mold walls is negligible which effectively 
acts as a no-slip boundary condition for the polymer phase. The second boundary condition does not allow either air or polymer to pass through the mold walls and the third boundary condition allows only air to pass through specified air outlets.

\subsection{Orientation equations}

The orientation equations can be written as

$$
\begin{aligned}
& \frac{\partial \mathbf{A}}{\partial t}+\mathbf{v} \cdot \nabla \mathbf{A}-c \mathbf{F}(\nabla \mathbf{v}, \mathbf{A})=\mathbf{0} \\
& \mathbf{A}\left(\Gamma_{\mathrm{i}}, t\right)=\mathbf{A}_{\mathrm{i}}
\end{aligned}
$$

where $\mathbf{F}(\nabla \mathbf{v}, \mathbf{A})$ is the model-specific term given by the right-hand sides of Eqs. (1), (2) and (3) for the standard Folgar-Tucker, the delayed Folgar-Tucker model and the RSC model, respectively. $\Gamma_{\mathrm{i}}$ is the inlet boundary of the simulation domain and $\mathbf{A}_{\mathrm{i}}$ is the orientation state prescribed at the inlet boundary. The orientation is determined in the entire simulation domain which consists of air as well as polymer. The distinction between the two phases is made by the pseudo-concentration variable $c$ which multiplies the model-specific term $\mathbf{F}(\nabla \mathbf{v}, \mathbf{A})$.

The orientation evolution equations in the form of Eq. (13) are discretized in time by means of the explicit Runge Kutta third order total variance diminishing (RK3-TVD) scheme [31] with a fixed time step $\Delta t$. The RK3-TVD scheme comprises three explicit Euler steps and two linear interpolation steps. Note that the pseudoconcentration and velocity are determined before the orientation at each time step and therefore values of $c^{n+1}$ and $\nabla \mathbf{v}^{n+1}$ are known at time $t^{n+1}$. The choice of RK3-TVD is made because it provides a stable solution, even with a relatively large time step as compared to the single step explicit methods, and computational time and memory requirements are low as compared to single step implicit methods [32].

The spatial discretization is performed using the discontinuous Galerkin finite element method (DGFEM) of Lesaint and Raviart [33]. In the DGFEM method, interpolation functions are defined locally on an element and the equations are solved separately on each element. The weak formulation for the 
solution of orientation equations on each element $\mathrm{K}$ with appropriate functional spaces for the test functions is given by: Find A such that for all admissible weighting functions $\Psi$

$$
\left\langle\frac{\partial \mathbf{A}}{\partial t}, \Psi\right\rangle_{\mathrm{K}}+\langle\mathbf{v} \cdot \nabla \mathbf{A}, \Psi\rangle_{\mathrm{K}}+\langle\mathbf{v} \cdot \mathbf{n}[\mathbf{A}], \Psi\rangle_{\Gamma_{\mathrm{K}, \mathrm{i}}}-\langle c \mathbf{F}(\mathbf{A}, \nabla \mathbf{v}), \Psi\rangle_{\mathrm{K}}=0
$$

where $\langle\mathbf{P}, \mathbf{Q}\rangle_{\mathrm{K}}$ and $\langle\mathbf{P}, \mathbf{Q}\rangle_{\Gamma_{\mathrm{K}, \mathrm{i}}}$ are proper $L^{2}$ inner products on the element domain $\Omega_{\mathrm{K}}$ and on the element inlet boundary $\Gamma_{\mathrm{K}, \mathrm{i}}$, respectively. The boundary integral is applied only on those boundaries of the element $\mathrm{K}$ for which $\mathbf{v} \cdot \mathbf{n}<0$, where $\mathbf{n}$ is the outward unit normal along the element boundary. $[\mathbf{A}]$ is the discontinuity across inter-element boundaries given by $[\mathbf{A}]=\mathbf{A}_{\Gamma_{\mathrm{K}, \mathrm{u}}}-\mathbf{A}_{\Gamma_{\mathrm{K}, \mathrm{e}}}$, where $\mathbf{A}_{\Gamma_{\mathrm{K}, \mathrm{u}}}$ is the value of $\mathbf{A}$ along the element inlet boundary $\Gamma_{K, \mathrm{i}}$ in the upstream element and $\mathbf{A}_{\Gamma_{\mathrm{K}, \mathrm{e}}}$ the value of $\mathbf{A}$ inside element $\mathrm{K}$ along the element inlet boundary $\Gamma_{\mathrm{K}, \mathrm{i}}$. If an inlet boundary $\Gamma_{\mathrm{K}, \mathrm{i}}$ of element $\mathrm{K}$ is along the inlet boundary of the domain, $\mathbf{A}_{\Gamma_{\mathrm{K}, \mathrm{u}}}=\mathbf{A}_{\mathrm{i}}$. In the last term of Eq. (14), the value of $c$ is determined at every integration point and $c=0$ is used if $c<0$, and $c=1$ is used if $c>1$ in order to eliminate nonphysical values of $c$ due to non-linear-interpolation. The advantage of the DGFEM formulation is reduced computational time and memory requirements as compared to the solution for the entire domain at once.

\subsection{Transport equation}

The evolution of the advancing front is captured by the pseudo-concentration method [24]. The pseudo-concentration variable $c$ evolves according to the transport equation

$$
\begin{aligned}
& \frac{\partial c}{\partial t}+\mathbf{v} \cdot \nabla c=0 \\
& c\left(\Gamma_{\mathrm{i}}, t\right)=1
\end{aligned}
$$

Eq. (15) is spatially discretized with the DGFEM [33] as described in section 5.2 with no modelspecific terms. Discretization in time is achieved by means of the RK3-TVD scheme [31]. The pseudoconcentration variable $c$ is initially set to zero in the entire simulation domain, indicating the presence of 
air. Only at the inlet domain boundary $\Gamma_{i}, c$ is specified as 1 , which indicates the presence of polymer at the inlet boundary of the domain.

\section{RESULTS}

Orientation predictions in the frontal region of a center-gated disk are compared with experimentally measured orientation data. The full thickness of the disk is simulated and all quantities are made dimensionless. The dimensions of the disk are shown in Fig. 1, non-dimensionalized by the half thickness $H$ of the disk. Simulation results are validated along the radial direction at three different heights, $z_{s} / H=$ $0.75, z_{s} / H=0.42$, and $z_{s} / H=0.08$, representative of the shell, transition and core layers, respectively. Measured orientation data in the frontal region of a disk as shown in Figs. 4-6 is used for experimental validation.

Mesh refinement is performed in both $r$ - and $z$-directions with the decoupled simulation of RSC model to ensure convergence of results. Fig. 7 shows the convergence of $A_{r r}$ along the radial direction in the shell and core layers. Five meshes are considered with elements in $r$ - and $z$-directions as 100x20, 200x30, 400x30, 400x40 and 600x40. The results are almost identical for the last three meshes.

Therefore, we select the 400x30 mesh for all our simulations in order to have sufficient accuracy as well as computational efficiency. In this mesh, the elements are $0.18 \times 0.33$ in non-dimensional units. As Fig. 7 shows, the $A_{r r}$ values near the interface adjust from the values in the polymer phase to equilibrium values in air phase over a very thin region covering the width of 2-3 elements in $r$-direction. The orientation on polymer side of the interface is induced primarily by velocity gradients in the frontal region. The equilibrium orientation on air side of the interface does not have a significant impact on orientation on the polymer side. A non-dimensional time step of 0.02 was selected for all simulations. Smaller time steps showed no significant difference in orientation predictions.

First, we discuss the effects of fountain flow on fiber orientation followed by an assessment of modified versions of the Folgar-Tucker model and effects of coupling on fiber orientation. 


\subsection{Effects of fountain flow on fiber orientation}

Fig. 8(a) shows the microscopic image of fiber footprints in the frontal region of a center-gated disk. $A_{r r}$ predictions of the decoupled RSC model are shown in Figs. 8(b), 8(c) and 8(d). The predicted shape of the front shown corresponds to $\mathrm{c}=0.5$ which denotes the melt-air interface. The value of $c$ drops from 1 to 0 over a distance of about two elements giving a sharp interface. The predicted front is almost semicircular in shape which matches qualitatively with the shape of the front observed in the microscopic image of the front.

Fiber footprints are shown as circular or elliptical cross sections in Fig. 8(a). Circular fiber cross sections denote fibers mainly oriented in the $\theta$-direction (large $A_{\theta \theta}$ component), perpendicular to the cross section shown in the figure. Large elliptical fiber cross sections correspond to fibers aligned in the radial direction (large $A_{r r}$ component). Simulations predict qualitatively key features observed in Fig. 8(a). Away from the front, a thin core layer is predicted having most of the fibers aligned in the $\theta$-direction while outer layers have significant fiber alignment in the radial direction. This layered structure is qualitatively similar to the structure observed in the microscopic image away from the front which shows circular cross sections in a thin layer near $z=0$ and large elliptical cross sections in the outer layers. The layered structure away from the front can be easily explained with the horizontal flowlines in the stationary reference frame (Fig. 8(b) and 8(c)). Along the flowlines in the core, the flow is dominated by extension which orients the fibers in the theta direction. Along the flowlines away from the core, the flow is dominated by shear which orients the fibers in the radial direction. Closer to the front, simulations predict a breakdown in the layered structure and the core layer broadens (as evident by a drop in $A_{r r}$ ) through the thickness of the disk. As depicted by the streamlines in stationary reference frame, $v_{z}$ jumps from almost zero to about 0.01 around $r / H=-2$ near the shell layer. Around that region, $A_{z z}$ increases to about $0.5-0.6$. Right near the interface, $A_{z z}$ is around 0.3 in the entire frontal region. This breakdown of the layered structure is also observed in the microscopic image which shows most of the fibers aligned in the $\theta$-direction near the front. Such phenomenon near the front can be explained with the flowlines in the moving reference frame (Fig. 8(d)). Fountain flow near the front transports the $\theta$-oriented fibers from the 
core layer to the outer layers resulting in a thickening of the core layer and a drop in $A_{r r}$ through the thickness of the disk.

Fig. 9 compares orientation values predicted by fountain flow and Hele-shaw flow simulations using the decoupled standard Folgar-Tucker model. Orientation predictions are compared with the experimental data of section 3. Fountain flow simulation shows relatively better qualitative agreement with the experimental data than Hele-Shaw flow simulation in all three layers. Hele-Shaw flow simulation, on the other hand, predicts steady values with no drop in orientation in any layer. However, there are significant quantitative differences in fountain flow predictions and the experimental data. The fountain flow simulation predicts a rapid evolution from high steady values in the lubrication region to low values near the front while experimental data shows gradual evolution. Fig. 9(a) shows orientation evolution along the shell layer. Fountain flow predicts a drop in orientation starting at around $r / H=-4$, followed by a rapid drop at approximately $r / H=-2.5$, then a slight increase between $r / H=-2$ and -1 , followed by a second rapid drop between $r / H=-1$ and the front. This is very similar to the trend seen in the experimental data. However, experimental data, shows a relatively gradual evolution over a greater distance. Experimental orientation data drops from $A_{r r}=0.69$ at $r_{s} / H=-14$ (experimental data available in a previous study [4]) to $A_{r r}=0.52$ at $r_{s} / H=-7.0$, followed by an increase between $r_{s} / H=-7.0$ and $r_{s} / H=-$ 5.6. The second drop in experimental orientation, which starts at distance approximately $r / H=-5.5$, is relatively slower than the drop seen in fountain flow predictions.

In the transition and core layers, as shown in Fig. 9(b) and 9(c) respectively, orientation predictions with fountain flow simulation also show improved qualitative agreement with the experimental data near the front while predictions with the Hele-Shaw flow approximation do not show any reduction in orientation. In the transition layer, predicted drop in orientation values with fountain flow simulations qualitatively matches the trend in experimental data. In the core layer, there is also good qualitative agreement between fountain flow predictions and the experimental data as both show a drop followed by a plateau very close to the front. $A_{r r}$ predictions very close to the front almost coincide with experimental data at $r_{s} / H=-0.7$ in both layers. However, as in the shell layer, there are significant quantitative 
differences between fountain flow predictions and experimental data. Evolution of orientation from the lubrication region to the front is not predicted correctly. In fountain flow simulation, the transition from high steady $A_{r r}$ values, representative of lubrication region, to low $A_{r r}$ values near the front starts only at approximately $r / H=-2$ in both layers while experimental data shows a relatively gradual transition starting as far back as $r_{s} / H=-7.0$.

\subsection{Assessment of delay in orientation evolution}

Fig. 10 shows fiber orientation predictions with the standard Folgar-Tucker, the delayed FolgarTucker and the RSC model using a decoupled simulation in the frontal region of a center-gated disk. In the shell layer, as shown in Fig. 10(a), evolution of orientation with the RSC model does not show a slowdown compared to the evolution predicted with the standard Folgar-Tucker model. Both the models predict a rapid drop in orientation starting approximately at $r / H=-2$. However, the delayed Folgar-Tucker model predicts a slowdown in orientation evolution with first gradual drop in orientation starting approximately at $r / H=-5$ and the second drop between $r / H=-2$ and $r / H=-1$. On the other hand, first a slight decrease in experimental data is seen in the region behind $r / H=-7$ and only the final drop from steady lubrication values to frontal values is observed in the region between $r / H=-5.6$ and the front. Even though the delayed Folgar-Tucker model improves orientation predictions in the frontal region, none of the models is able to fully capture the slow evolution of orientation in the frontal region.

In transition and core layers, shown in Figs. 10(b) and 10(c), predictions of all three models almost coincide with a drop in orientation starting at approximately $r / H=-2$. Plateau values at the front are in good agreement with experimental data at $r_{s} / H=-0.7$ in both layers, especially with two modified forms of the Folgar-Tucker model. However, none of the modified versions is able to achieve the slowdown in orientation evolution from steady lubrication values to frontal values seen in experimental data in both layers. The predicted transition in orientation from the lubrication region to the front starts only at approximately $r / H=-2$. Experimental data, on the other hand, starts to decline much earlier, at $r_{s} / H \sim-7$. At first sight, it seems that a small $\alpha$ value could lead to larger frontal regions as observed in the 
experiments. However, very small $\alpha$ values would slow down the evolution in the entry region too much, leading to a considerably larger $A_{r r}$ value at $r / H=-8$ in the core region. A steady value of $A_{r r}$ would not be reached then, as observed in the experiments in [26].

\subsection{Effects of coupling on velocity and orientation in frontal region}

The effects of coupling on orientation predictions in the frontal region were investigated by comparing coupled and decoupled simulations with experimental orientation values. Coupling parameters determined from rheological fitting as well as theoretical calculations based on Dinh-Armstrong theory were considered.

In order to understand the difference in orientation predictions between decoupled and coupled simulations, it would be suitable to look at velocity profiles first. Fig. 11 shows velocity predictions along three different layers in decoupled and coupled simulations using the RSC model with $C_{I}=0.0112$. Decoupled simulations $\left(r_{s t r}=0\right)$ have the Newtonian velocity profile. As we get closer to the front, $v_{r}$ shows an increase in the shell layer $\left(z_{s} / H=0.75\right)$ and a decline in the layers near the core $\left(z_{s} / H=0.42\right.$ and $\left.z_{s} / H=0.08\right)$. The transverse velocity $\left(v_{z}\right)$, on the other hand, shows a peak in all three layers very close to the front. In coupled simulations, $v_{r}$ does not show a significant change compared to the Newtonian velocity profile. However, non-zero $v_{z}$ profiles are observed over a larger region $-5 \leq r / H \leq 0$ for the coupled simulations. Therefore fluid starts flowing towards the wall further away from the front leading to a larger region of low $A_{r r}$ values, typical for the frontal region. In addition, the $v_{z}$ profile for the coupled simulations is smoother, leading to a more gradual evolution of $A_{r r}$ values in the frontal region.

Fig. 12 shows profiles of $A_{r r}$ along three different layers in coupled and decoupled simulations using the RSC model with $C_{I}=0.0112$. In all three layers, orientation predictions in coupled simulation with $\boldsymbol{k}_{\text {str }}$ $=6.18$ are relatively closer to orientation predictions with $k_{t r}^{\zeta}=0$ (decoupled simulation) while at $k_{\text {tr }}^{\zeta}=$ 37, evolution in orientation is slower compared to decoupled simulation. Differences in orientations predictions between coupled and decoupled simulations could be attributed to the difference in the velocity field between coupled and decoupled simulations. In coupled simulations, velocity transition 
between the lubrication and the frontal region is more gradual and starts early compared to decoupled simulation, as indicated by broader peaks in $v_{z}$ profiles. This indicates that the frontal flow region covers a larger distance behind the front in coupled simulations and could explain gradual decline of $A_{r r}$ seen in coupled simulations compared to decoupled simulation. However, neither coupled nor decoupled simulation is slow enough to capture the slowdown observed in experimental orientation near the front. Fig. 13 shows profiles of $A_{z z}$ and $A_{\theta \theta}$ along three different layers in coupled and decoupled simulations using the RSC model with $C_{I}=0.0112 . A_{z z}$ predictions are in close proximity to experimentally measured values in all three layers except in the region between the front and $\mathrm{r} / \mathrm{H} \sim-2 . A_{\theta \theta}$ shows a similar trend as $A_{r r}$ except that $A_{\theta \theta}$ increases near the front Coupled simulations, especially at $\boldsymbol{\zeta}_{t r}=37$ show gradual transition compared to decoupled simulation. However for both $A_{z z}$ and $A_{\theta \theta}$, the slowdown is not enough to match the slow evolution seen in experimental data.

\subsection{Effects of $C_{I}$ on orientation in coupled simulations}

Simulations with the RSC model and two different values of $C_{I}$ were also compared with experimental orientation data. Two values of $C_{I}$ were considered, $C_{I}=0.112$ fitted to rheological data in startup of shear by Mazahir et al. [4], and $C_{I}=0.001$ used by Chung and Kwon [2]. In both cases, coupled simulations were performed with $\zeta_{s t r}=37$. Model predictions are compared with experimental data along the entire flow length of the disk (from the gate upto $r / H=-0.7$ behind the front) in order to assess the effects of $C_{I}$ in both lubrication and frontal regions. Fig. 14 shows $A_{r r}$ predictions along the entire flow length and a magnified view of the front. Coupled simulations at lower level of $C_{I}(0.001)$ show relatively high level of non-physical oscillations. Differences between the two $C_{I}$ values are primarily observed in the shell layer. On comparison with experimental data in the lubrication region along the shell layer $\left(z_{s} / H\right.$ $=0.75), C_{I}=0.0112$ shows a relatively good orientation prediction while $C_{I}=0.001$ predicts higher flowdirection orientation. This is expected as a lower $C_{I}$ allows for shear flow near the walls to dominate and fibers orient along the direction of flow. However, as we get closer to the front, $C_{I}=0.001$ in coupled simulation shows a greater decline in orientation that starts much earlier (around $r / H=-17$ ) compared to 
$C_{I}=0.0112$. It must be noted that $C_{I}=0.0112$ was fitted to rheological experiments under startup of shear and therefore may be suitable for the lubrication region which is dominated by shear flow. However, as we get closer to the front, extensional flow dominates and therefore, startup of shear may not be the most appropriate method for determining model parameters. There may be a need to fit model parameters under extensional flow and use a combination of parameters from two methods for the fountain flow region. In the transition and core layers, $A_{r r}$ predictions with $C_{I}=0.0112$ and $C_{I}=0.001$ are very similar in the lubrication region. However, in the frontal region of the transition layer, orientation starts to drop relatively earlier when $C_{I}=0.001$ is used.

$A_{z z}$ predictions with $C_{I}=0.001$ are in very good agreement with experimental data in the lubrication region in all three layers. However, closer to the front, coupled simulations show non-physical oscillations, especially when $C_{I}=0.001$ is used, as shown in Fig. 15. The transition layer has a similar profile as the core layer and is omitted for the sake of brevity. $A_{\theta \theta}$ predictions show a very similar trend to $A_{r r}$ in all three layers, except that $A_{\theta \theta}$ profiles are almost a mirror image of $A_{r r}$ profiles.

\section{CONCLUSIONS AND DISCUSSION}

An experimental study of fiber orientation the frontal region of a $30 \mathrm{wt} \%$ short glass fiber injectionmolded center-gated disk was carried out in order to assess evolution of orientation in the frontal region. The effects of fountain flow on orientation are seen in all three layers with a significant drop in flowdirection orientation from steady values representative of the lubrication region to substantially lower values near the front. Accurate numerical techniques have been used to capture the rapid evolution of the fiber orientation in the relatively small frontal region. Accurate prediction of the orientation in the frontal region is, however, important because it governs orientation that is frozen in near the walls when the mold is cooled. As a result, changes in orientation in the frontal region will affect the fiber orientation along the entire walls. In addition, for small parts or small structures in a larger part, the frontal flow region can be much more significant, and being able to understand the flow behavior and orientation at the front becomes much more important. 
Orientation predictions with the RSC model in coupled simulations were compared with experimentally measured orientation values. RSC model parameters were fitted to rheological data in startup of shear. When the transverse component of velocity was included in the simulation to predict fountain flow near the front, the frontal region covered a larger distance behind the front than in decoupled simulations. This is a direct consequence of the predicted velocity profiles. The coupled RSC model was able to slightly slow down the evolution of orientation in the frontal region. However, the slowdown was not enough to match the even slower evolution observed in experimental data. Two other effects that could play a role in the discrepancies observed between the experiments and simulations are the presence of small voids near the front and a relatively small thickness of the disk compared to the fiber length. Voids seem to affect the orientation very locally as can be observed in Fig. 8, where some changes in fiber orientation can be noticed for the fibers immediately surrounding the voids. A little further away from the voids, however, the values of the orientation don't seem to be very sensitive to the presence of small holes and the measurements seem to be reproducible. In addition, even though the locations of the voids were different, the orientation measurements for the two different disks did not show significant differences when bins were chosen so that they did not contain voids. A relatively small thickness of the mold could possibly affect the rotation of the fibers. In simple shear flow, however, fiber orientation became independent of the gap width for a thickness of 3 fiber lengths. The key reason for this seems that, the fibers mainly stay oriented in the $r \theta$ plane for the rheometrical flow so that the effective length of the fibers in the direction of the thickness is considerably smaller than the fiber length of 364 $\mu \mathrm{m}$ [19]. Also for the center-gated disk the fibers mainly stay in the $r \theta$ plane, and for $A_{z z}$ the simulations are in qualitative agreement with the experimental values. In our relatively narrow and short center-gated disk we used for this study, a possible reason is that the $\theta \theta$ component of the velocity gradient may still be large enough to keep fibers oriented in the $r \theta$ plane. For larger molds, however, it may be important to have a larger mold thickness because the $\theta \theta$ component of the velocity gradient is proportional to $1 / r^{2}$ and a more significant orientation in the gapwise direction may be present at large radial distances $r / H$. 
Coupled simulations were also carried out with two different values of $C_{I}$ using the RSC model. In the lubrication region of the shell layer, $A_{r r}$ predictions were closer to experimental data with $C_{I}=0.0112$ compared to predictions with $C_{I}=0.001$. However, in the frontal region, $C_{I}=0.001$ showed relatively better predictions compared to $C_{I}=0.0112$. The larger $C_{I}$ value $(0.0112)$, which was determined from rheological experiments in startup of shear seems to provide better predictions in the shear-dominated lubrication region but may not be suitable for extension-dominated frontal region. Therefore it is recommended that rheological experiments in extensional flow be conducted to determine $C_{I}$ for the frontal region.

\section{ACKNOWLEDGEMENTS}

The authors wish to gratefully acknowledge the financial support for this work from the Department of Energy and the National Science Foundation through grants No. DMI-0521918 and No. CMMI0853537, respectively. The authors also like to thank Sabic Americas Inc. for providing the Valox 420 material used in experimental studies in this work. The access to polishing and microscopic facilities at the Material Science and Engineering Department and to the microscope at Institute of Critical Technology and Applied Sciences (ICTAS) at Virginia Tech is gratefully acknowledged. Syed Mazahir gratefully acknowledges the support provided by ICTAS through the Doctoral Scholars program.

Gregorio M. Vélez-García would like to thank NSF-IGERT for funding through grant No. DGE-0548783, the Macromolecular Science and Infrastructure Engineering, and the University of Puerto RicoMayagüez.

\section{REFERENCES}

[1] W. Rose, Fluid-Fluid Interfaces in Steady Motion, Nature 191 (1961) 242-243.

[2] D.H. Chung, T.H. Kwon, Numerical studies of fiber suspensions in an axisymmetric radial diverging flow: the effects of modeling and numerical assumptions, Journal of Non-Newtonian Fluid Mechanics 107 (2002) 67-96.

[3] J.M. Park, T.H. Kwon, Nonisothermal transient filling simulation of fiber suspended viscoelastic liquid in a center-gated disk, Polymer Composites 32 (2011) 427-437. 
[4] S.M. Mazahir, G.M. Vélez-García, P. Wapperom, D.G. Baird, Evolution of fiber orientation in radial direction in a center-gated disk: Experiments and simulation, Compos. Part A-Appl. S. 51 (2013) 108-117.

[5] R.S. Bay, C.L. Tucker, Fiber orientation in simple injection moldings. Part II: Experimental results, Polymer Composites 13 (1992) 332-341.

[6] G.M. Vélez-García, S.M. Mazahir, P. Wapperom, D.G. Baird, Simulation of Injection Molding Using a Model with Delayed Fiber Orientation, International Polymer Processing 26 (2011) 331339.

[7] F. Folgar, C.L. Tucker, Orientation behavior of fibers in concentrated suspensions, Journal of reinforced plastics and composites 3 (1984) 98-119.

[8] R.S. Bay, C.L. Tucker, Fiber orientation in simple injection moldings. Part I: Theory and numerical methods, Polymer Composites 13 (1992) 317-331.

[9] B.E. Verweyst, C.L. Tucker, Fiber Suspensions in Complex Geometries: Flow/Orientation Coupling, Canadian Journal of Chemical Engineering 80 (2002) 1093-1106.

[10] K.H. Han, Y.T. Im, Numerical simulation of three-dimensional fiber orientation in injection molding including fountain flow effect, Polymer Composites 23 (2002) 222-238.

[11] J. Ko, J.R. Youn, Prediction of fiber orientation in the thickness plane during flow molding of short fiber composites, Polymer Composites 16 (1995) 114-124.

[12] M. Gupta, K.K. Wang, Fiber orientation and mechanical properties of short-fiber-reinforced injection-molded composites: Simulated and experimental results, Polymer Composites 14 (1993) 367-382.

[13] S.T. Chung, T.H. Kwon, Coupled analysis of injection molding filling and fiber orientation, including in-plane velocity gradient effect, Polymer Composites 17 (1996) 859-872.

[14] S.T. Chung, T.H. Kwon, Numerical simulation of fiber orientation in injection molding of shortfiber-reinforced thermoplastics, Polymer Engineering and Science 35 (1995) 604-618.

[15] M. Vincent, E. Devilers, J.F. Agassant, Fibre orientation calculation in injection moulding of reinforced thermoplastics, Journal of Non-Newtonian Fluid Mechanics 73 (1997) 317-326.

[16] S. Ranganathan, S.G. Advani, A simultaneous solution for flow and fiber orientation in axisymmetric diverging radial flow, Journal of Non-Newtonian Fluid Mechanics 47 (1993) 107136.

[17] G.B. Jeffery, The Motion of Ellipsoidal Particles Immersed in a Viscous Fluid, in Proceedings of the Royal Society of London, Series A, 1922: The Royal Society.

[18] M. Sepehr, G. Ausias, P.J. Carreau, Rheological properties of short fiber filled polypropylene in transient shear flow, Journal of Non-Newtonian Fluid Mechanics 123 (2004) 19-32.

[19] A.P.R. Eberle, G.M. Vélez-García, D.G. Baird, P. Wapperom, Fiber orientation kinetics of a concentrated short glass fiber suspension in startup of simple shear flow, Journal of Non-Newtonian Fluid Mechanics 165 (2010) 110-119.

[20] A.P.R. Eberle, D.G. Baird, P. Wapperom, G.M. Vélez-García, Using transient shear rheology to determine material parameters in fiber suspension theory, Journal of Rheology 53 (2009) 685-705.

[21] J. Wang, J.F. O'gara, C.L. Tucker, An objective model for slow orientation kinetics in concentrated fiber suspensions: Theory and rheological evidence, J. Rheol. 52 (2008) 1179.

[22] H.M. Huynh, Improved fiber orientation predictions for injection-molded composites, 2001, Universty of Illinois - Urbana Champaign: Urbana, Ill.

[23] B.E. VerWeyst, C.L. Tucker, P.H. Foss, J.F. O'Gara, Fiber orientation in 3-D injection molded features: prediction and experiment, International Polymer Processing 4 (1999) 409-420.

[24] G. Haagh, F. Van De Vosse, Simulation of three-dimensional polymer mould filling processes using a pseudo-concentration method, International Journal for Numerical Methods in Fluids 28 (1998) 1355-1369.

[25] J.H. Phelps, C.L. Tucker III, An anisotropic rotary diffusion model for fiber orientation in shortand long-fiber thermoplastics, Journal of Non-Newtonian Fluid Mechanics 156 (2009) 165-176. 
[26] G.M. Vélez-García, P. Wapperom, D.G. Baird, A.O. Aning, V. Kunc, Unambiguous orientation in short fiber composites over small sampling area in a center-gated disk, Composites Part A: Applied Science and Manufacturing 43 (2012) 104-113.

[27] A.R. Clarke, C.N. Eberhardt, Microscopy Techniques for Materials Science: CRC Press. Place 2002.

[28] D. Chung, T.H. Kwon, Invariant-based optimal fitting closure approximation for the numerical prediction of flow-induced fiber orientation, J. Rheol. 46 (2002) 169.

[29] S.M. Dinh, R.C. Armstrong, A Rheological Equation of State for Semiconcentrated Fiber Suspensions, Journal of Rheology 28 (1984) 207-227.

[30] P.C. Wu, C.F. Huang, C.G. Gogos, Simulation of the mold-filling process, Polymer Engineering and Science 14 (1974) 223-230.

[31] C.-W. Shu, S. Osher, Efficient implementation of essentially non-oscillatory shock-capturing schemes, Journal of Computational Physics 77 (1 988) 439-471.

[32] W.R. Hwang, W.-Y. Kim, S.H. Kang, S.J. Kim, Direct simulations on 2D mold-filling processes of particle-filled fluids, Korea-Australia Rheology Journal 21 (2009) 193-200.

[33] P. Lesaint, P.A. Raviart, On a Finite Element Method for Solving the Neutron Transport Equation, in Mathematical Aspects of Finite Elements, C.d. Boor, Editor, Academic Press, New York, 1974, p. 89-123.

[34] S.M. Mazahir, G.M. Vélez-García, P. Wapperom, D. Baird, Evolution of fibre orientation in radial direction in a center-gated disk: Experiments and simulation, Composites Part A: Applied Science and Manufacturing 51 (2013) 108-117. 


\section{FIGURES}

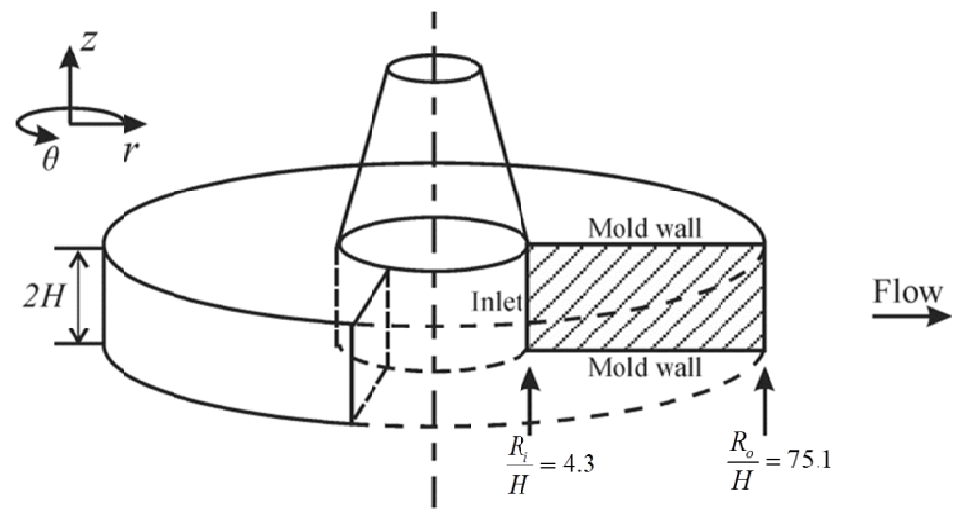

(a)

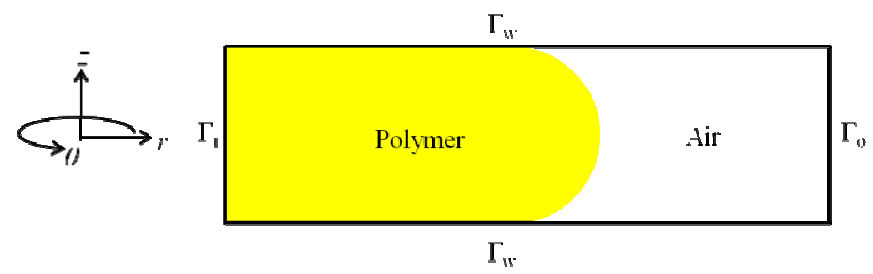

(b)

Figure 1: Center-gated disk with dimensions normalized by the half thickness $H$ of the disk (a) and the simulation domain and boundaries (b). 


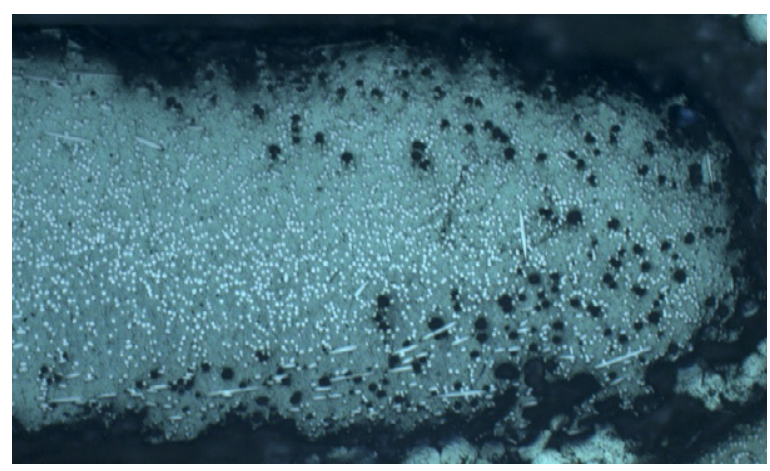

(a)

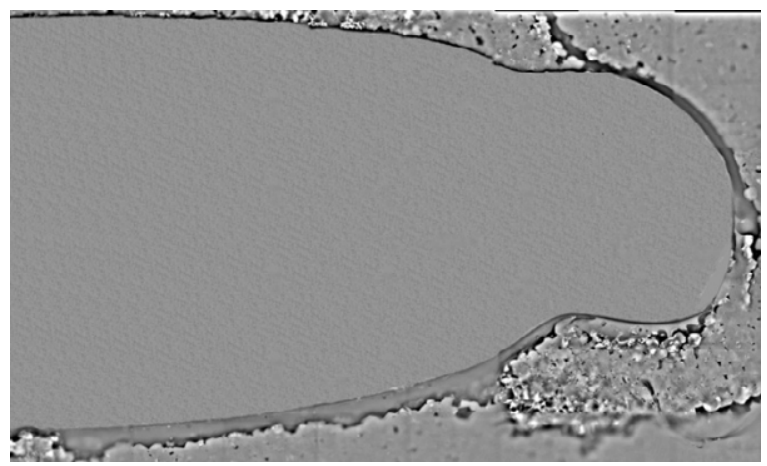

(b)

Figure 2: Microscopic image at $5 \mathrm{X}$ zoom of the frontal region of a center-gated disk of (a) PBT/30 wt\% short glass fiber suspension and (b) pure PBT. 


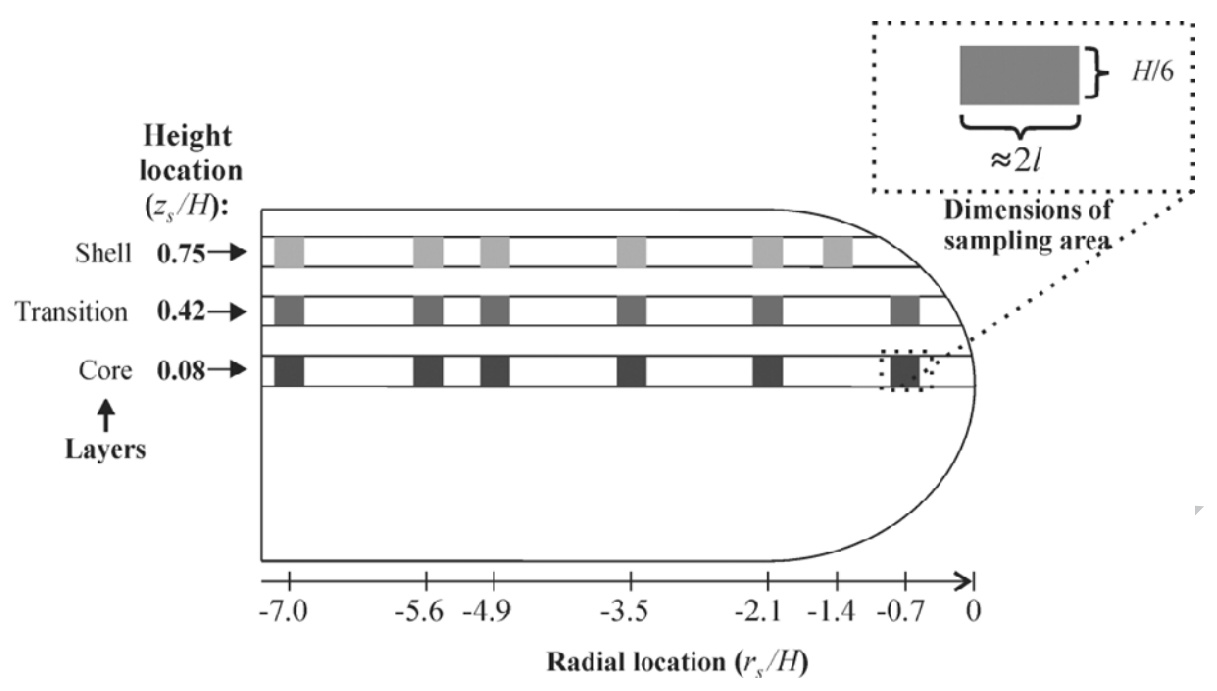

Figure 3: Radial locations in the frontal region of a center-gated disk selected for measurement of fiber orientation. Radial locations are shown in terms of non-dimensional distance from the front. Insert shows the dimensions of the sampling area.

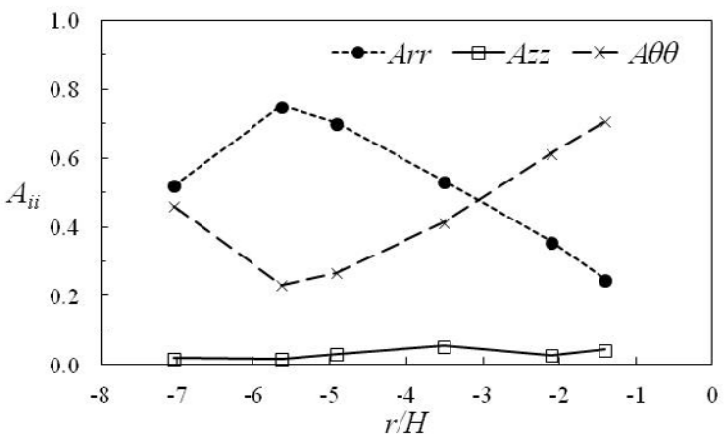

(a)

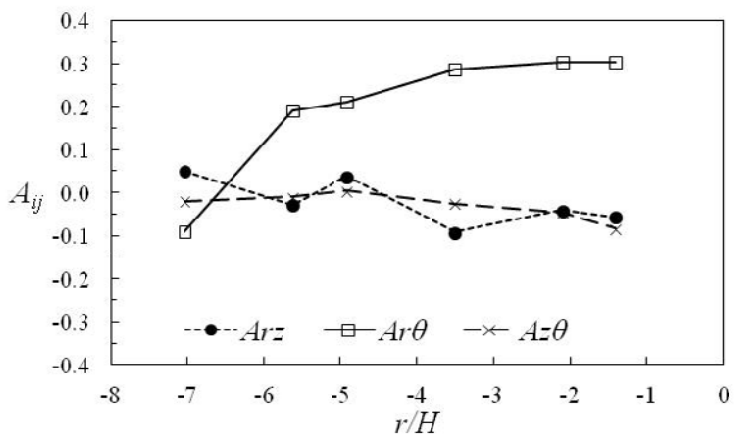

(b)

Figure 4: Orientation profile of (a) diagonal $A_{i i}$ and (b) off-diagonal $A_{i j}$ components along the shell layer $\left(z_{s} / H=0.75\right)$ in the frontal region of a center-gated disk. 


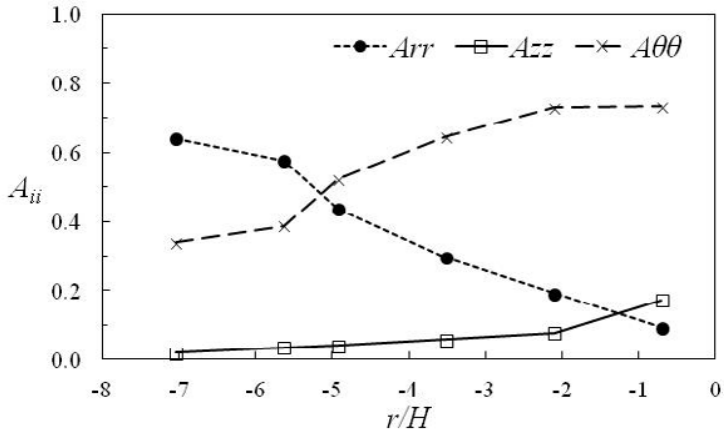

(a)

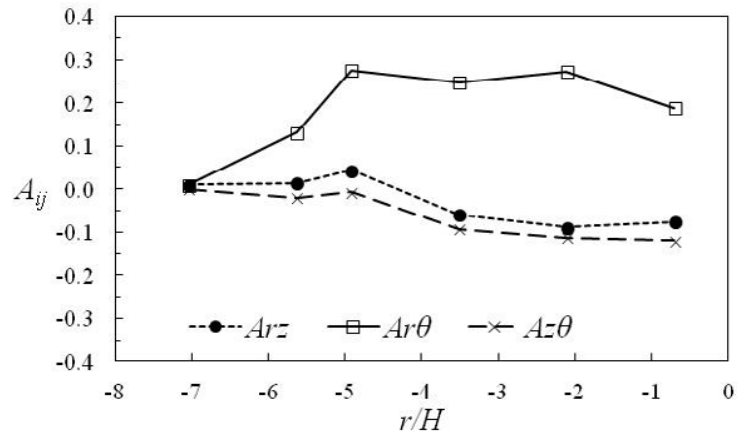

(b)

Figure 5: Orientation profile of (a) diagonal $A_{i i}$ and (b) off-diagonal $A_{i j}$ components along the transition layer $\left(z_{s} / H=0.42\right)$ in the frontal region of a center-gated disk.

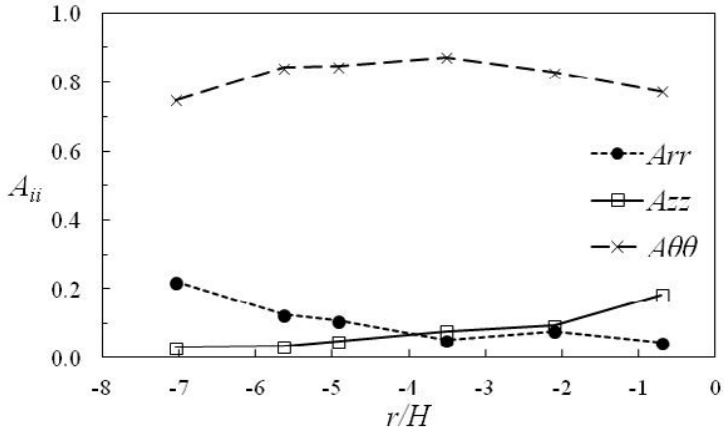

(a)

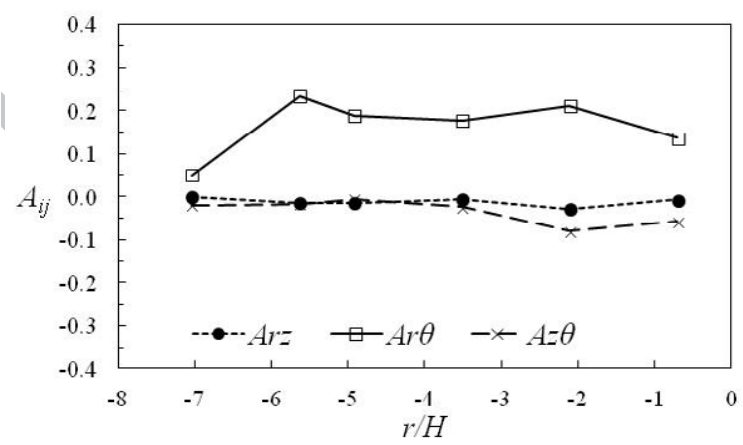

(b)

Figure 6: Orientation profile of (a) diagonal $A_{i i}$ and (b) off-diagonal $A_{i j}$ components along the core layer $\left(z_{s} / H=0.08\right)$ in the frontal region of a center-gated disk. 


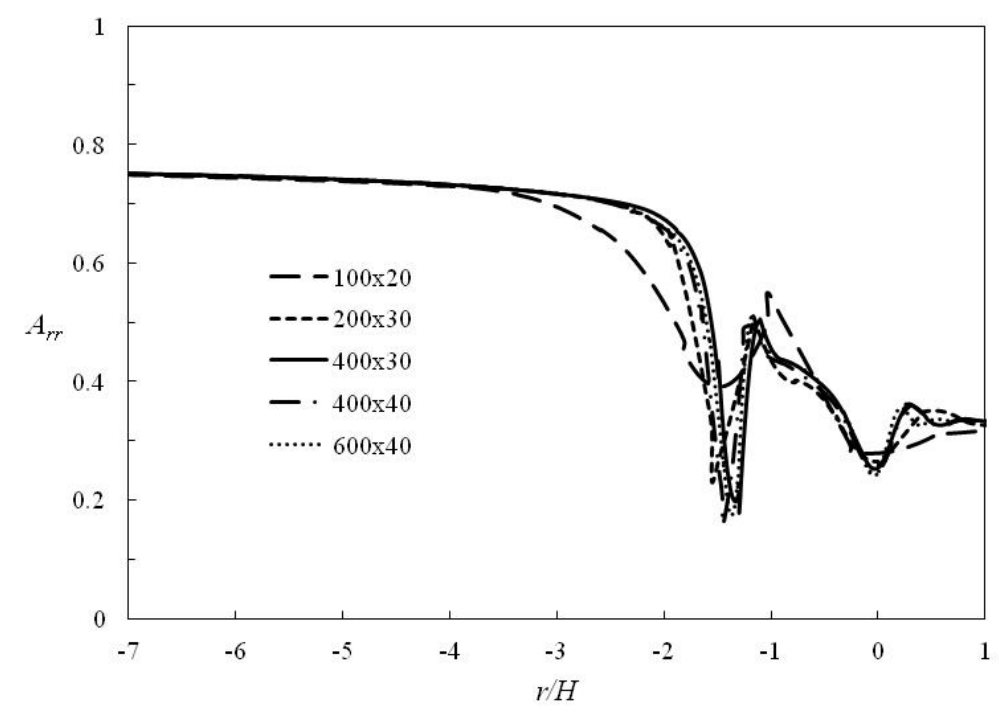

(a)

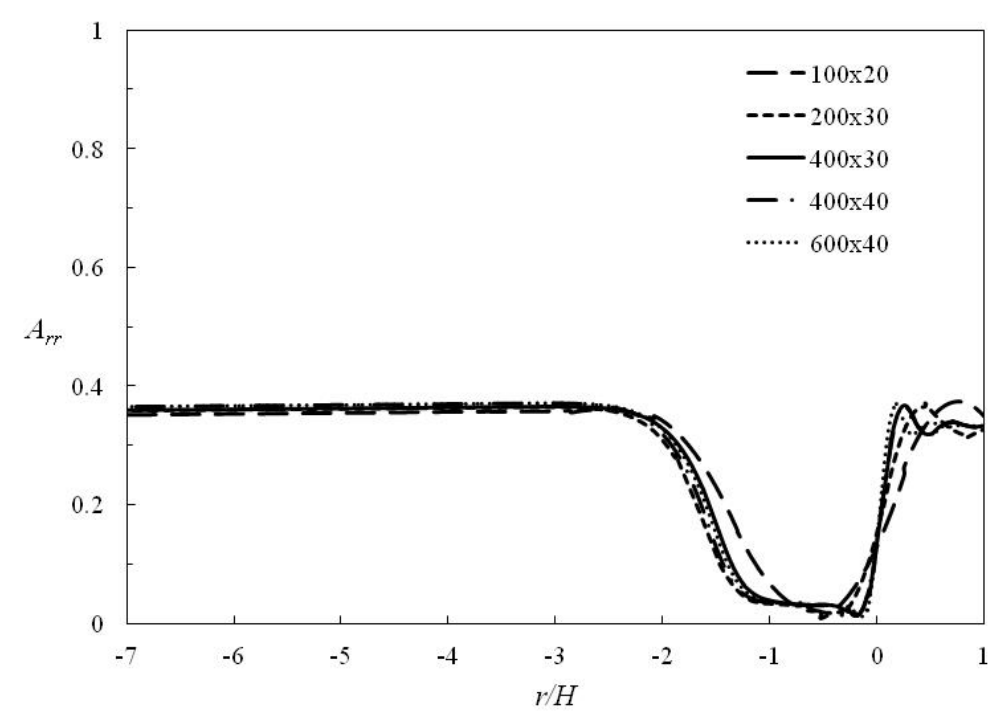

(b)

Figure 7: Convergence of $A_{r r}$ predicted by the RSC model in decoupled fountain flow simulation.

Fives meshes are considered with mesh refinement in both $r$-and $z$-directions. $A_{r r}$ predictions are shown at (a) $z_{s} / H=0.75$, (b) $z_{s} / H=0.08$. 


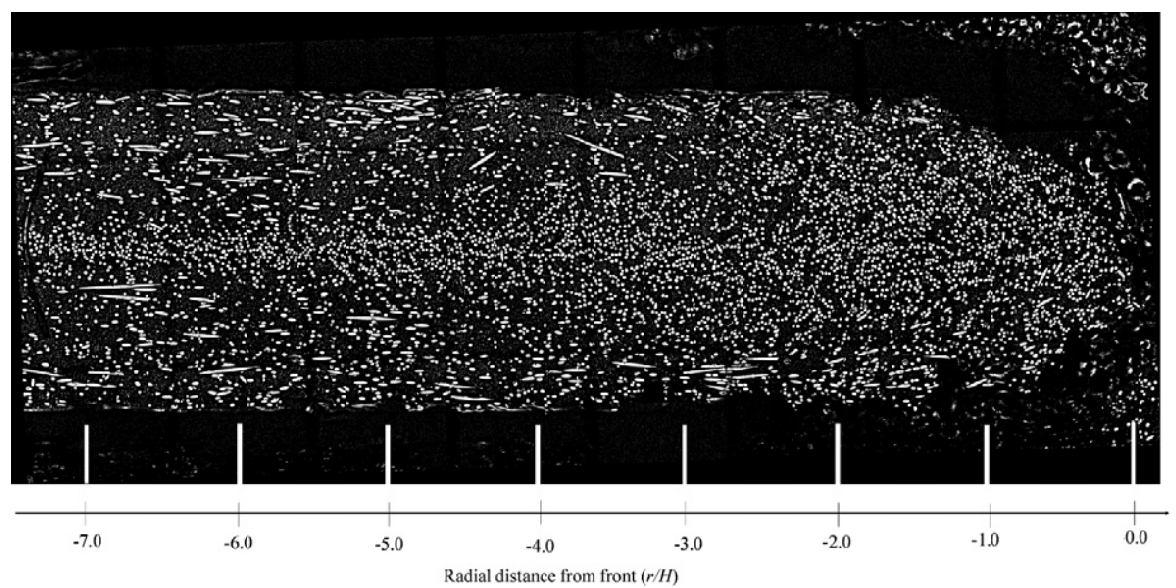

(a)
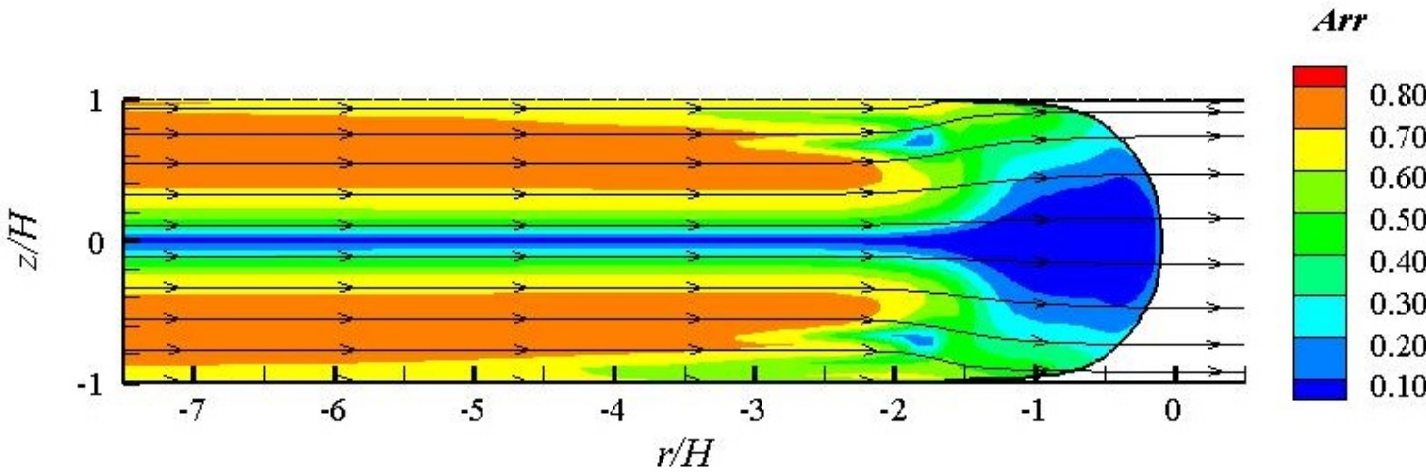

(b)
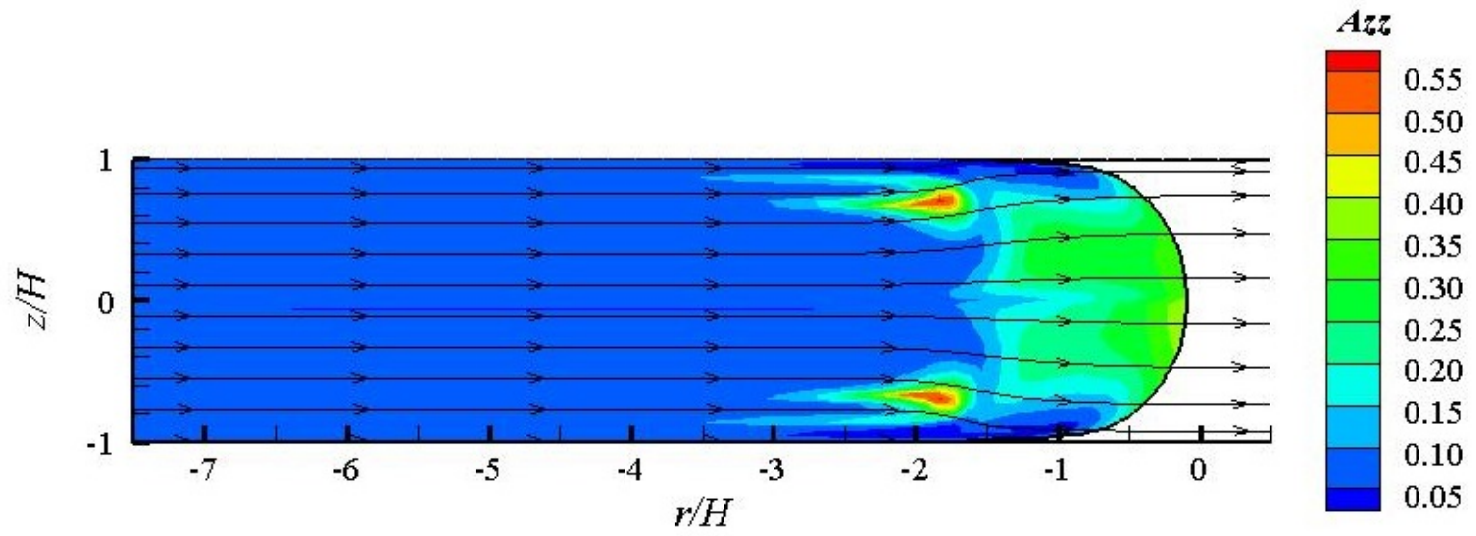

(c) 


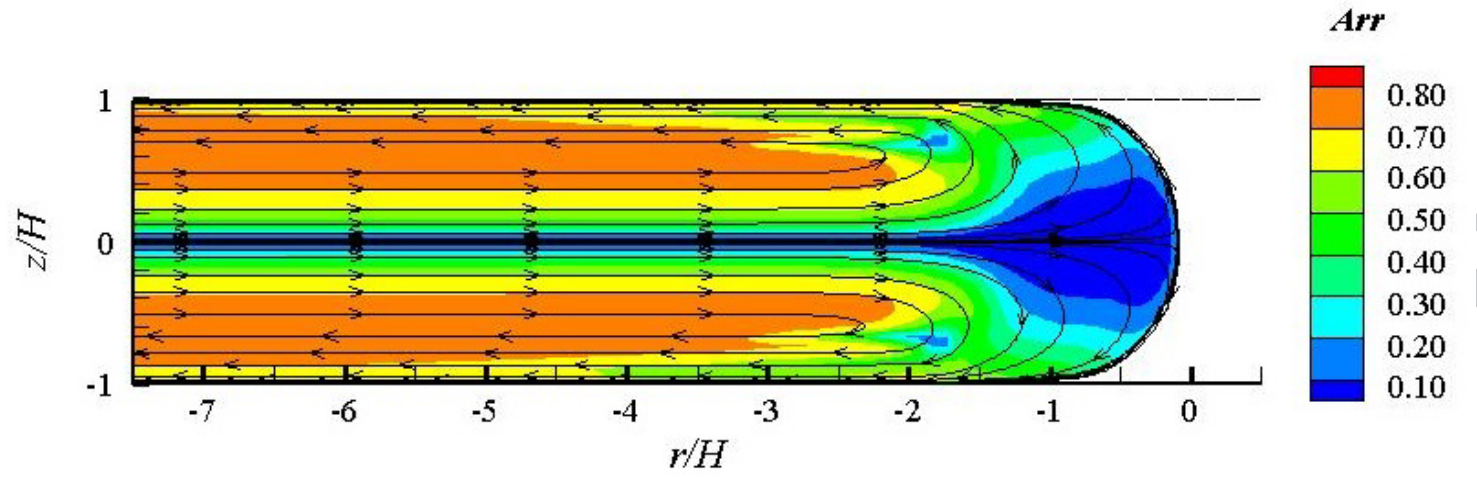

(d)

Figure 8: Comparison of (a) microscopic image at 20X zoom showing fiber cross sections and contour plots of predicted orientation for the RSC model in a decoupled simulation in the frontal region of a center-gated disk and streamlines for (b) $A_{r r}$ in a stationary reference frame, (c) $A_{z z}$ in a stationary reference frame, and (d) $A_{r r}$ in a moving reference frame attached to the tip of the front. 


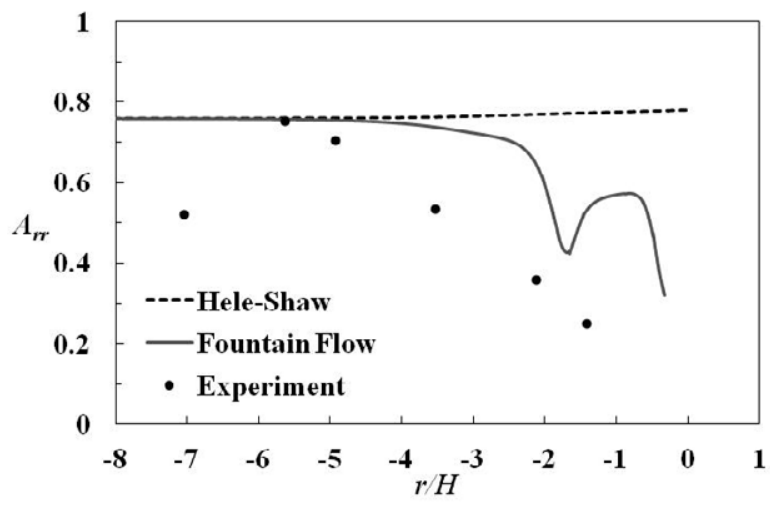

(a)

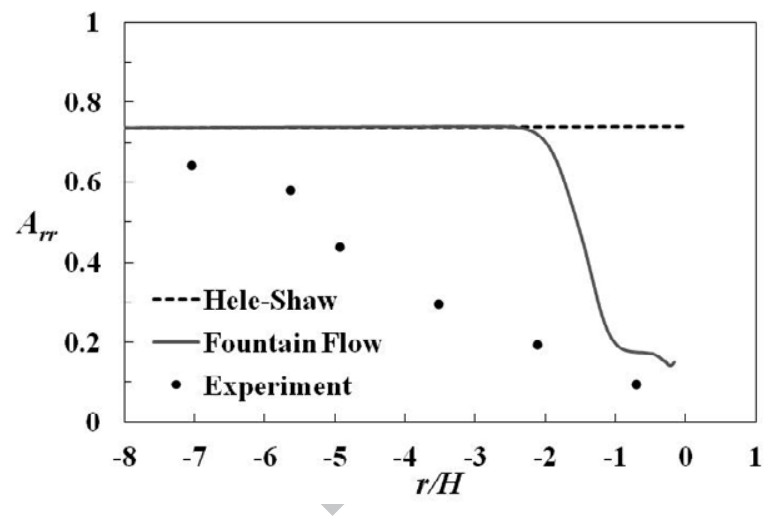

(b)

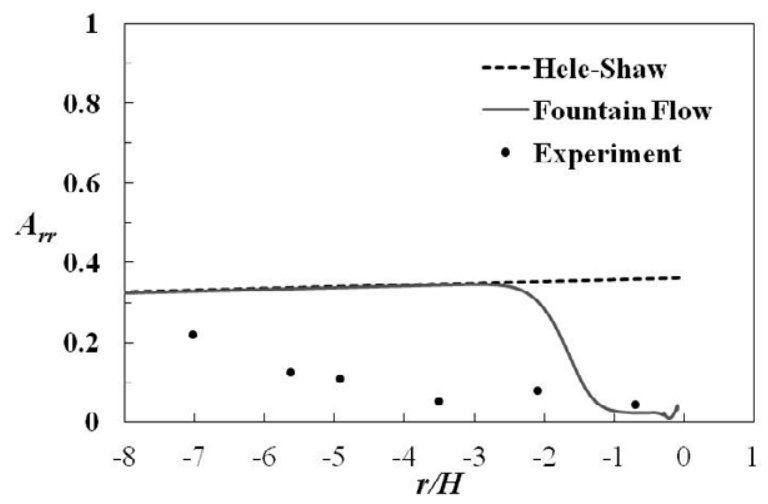

(c)

Figure 9: $A_{r r}$ predictions using the standard Folgar-Tucker model with Hele-Shaw flow approximation and fountain flow simulation in a decoupled scheme, compared with experimentally measured values at (a) $z_{s} / H=0.75$, (b) $z_{s} / H=0.42$, and (c) $z_{s} / H=0.08$. 


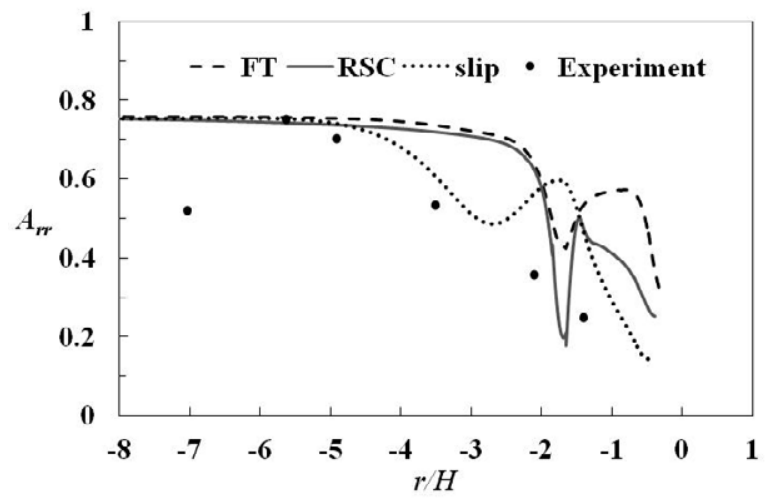

(a)

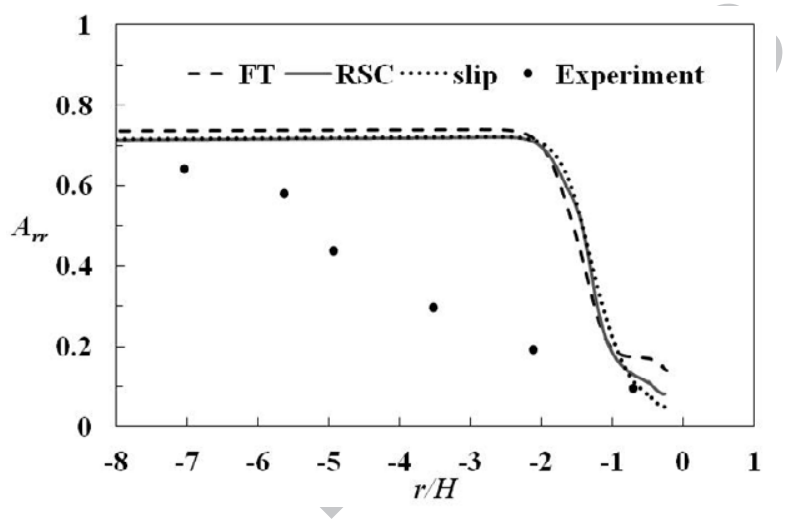

(b)

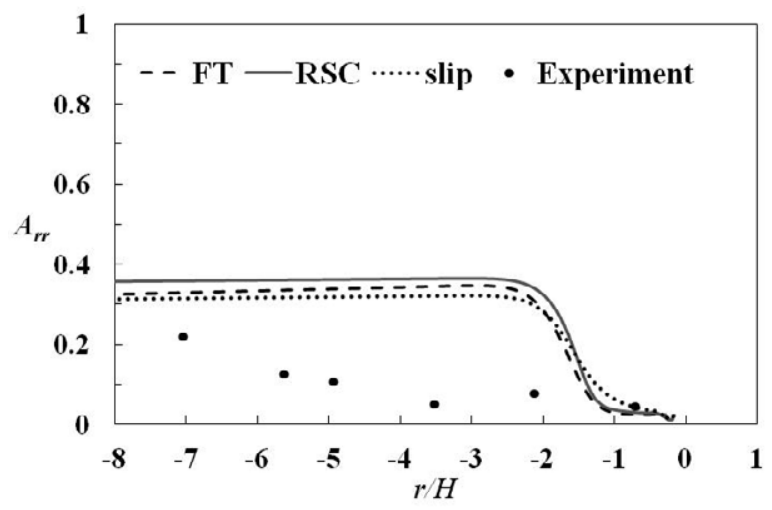

(c)

Figure 10: Comparison of $A_{r r}$ predictions of the standard Folgar-Tucker, RSC and slip models using decoupled fountain flow simulation with experimentally measured values at (a) $z_{s} / H=0.75$, (b) $z_{s} / H=$ 0.42 , and $(\mathrm{c}) z_{s} / H=0.08$. 


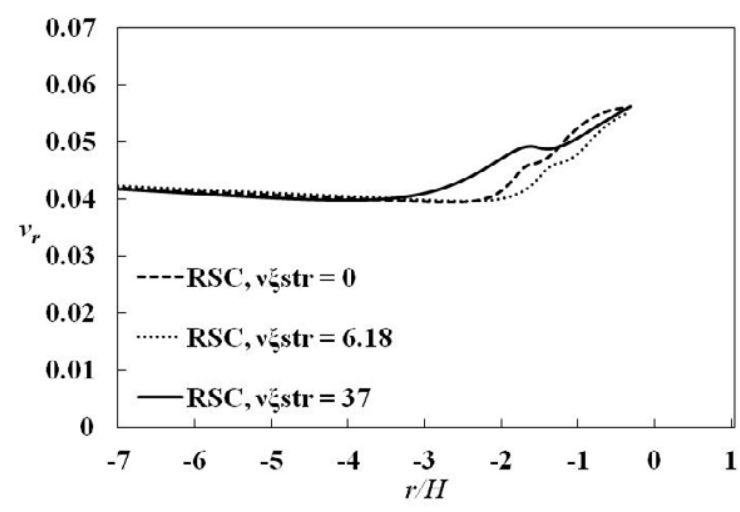

(a)

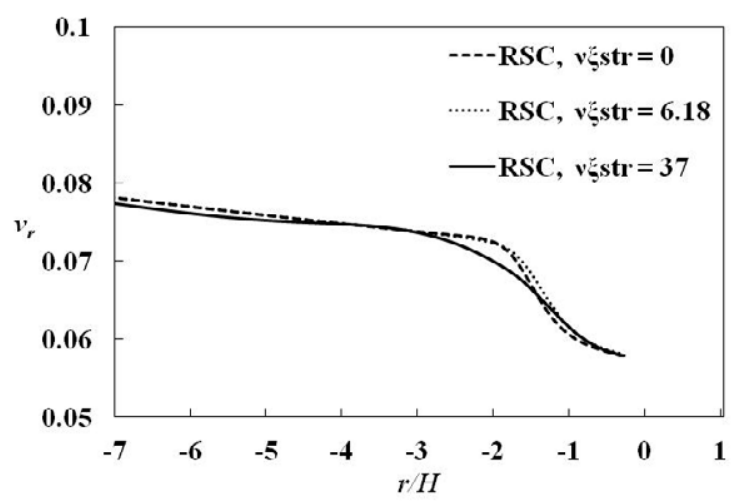

(c)

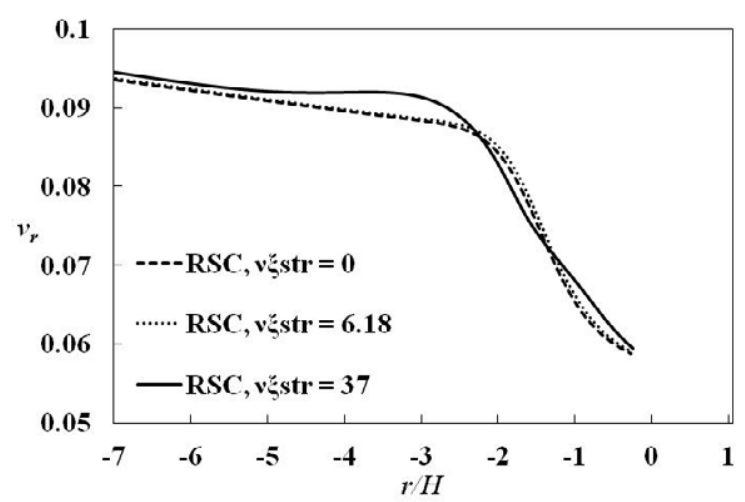

(e)

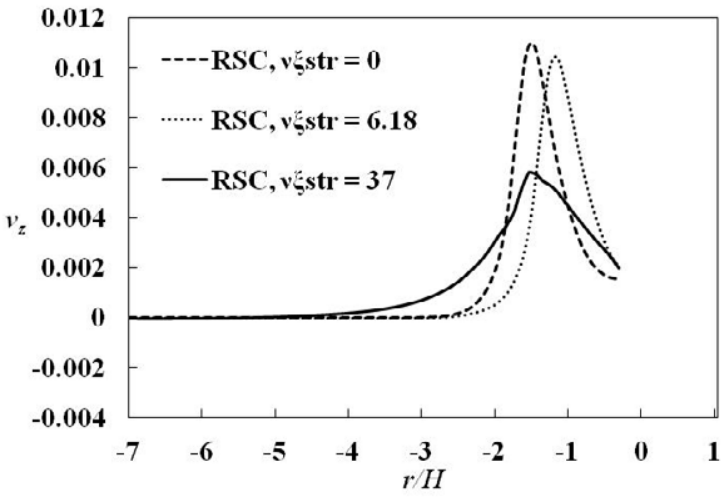

(b)

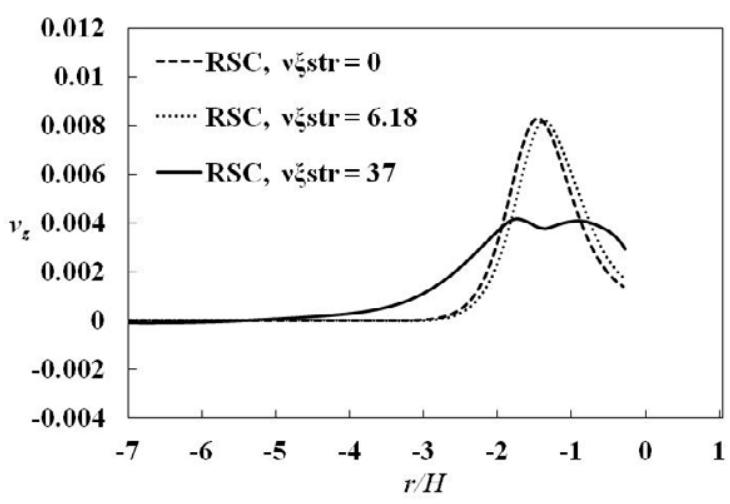

(d)

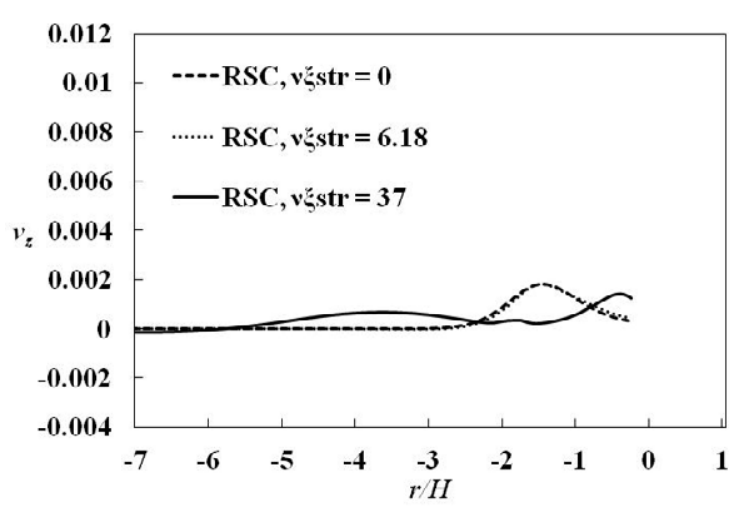

(f)

Figure 11: Velocity predictions in decoupled and coupled simulations using the RSC model and $C_{I}=$ 0.0112 at (a), (b) $z_{s} / H=0.75$, (c), (d) $z_{s} / H=0.42$, and (e), (f) $z_{s} / H=0.08$. 


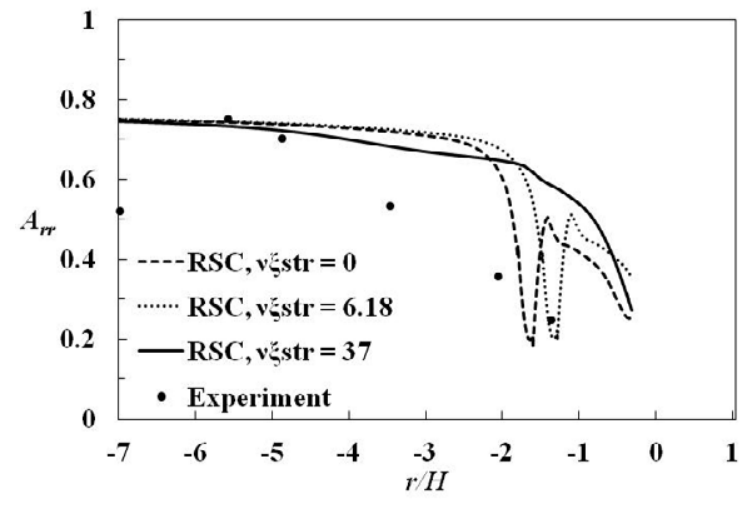

(a)

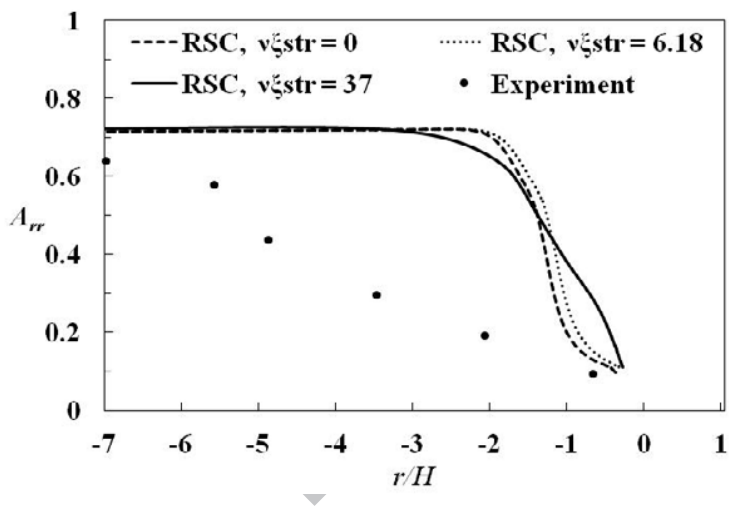

(b)

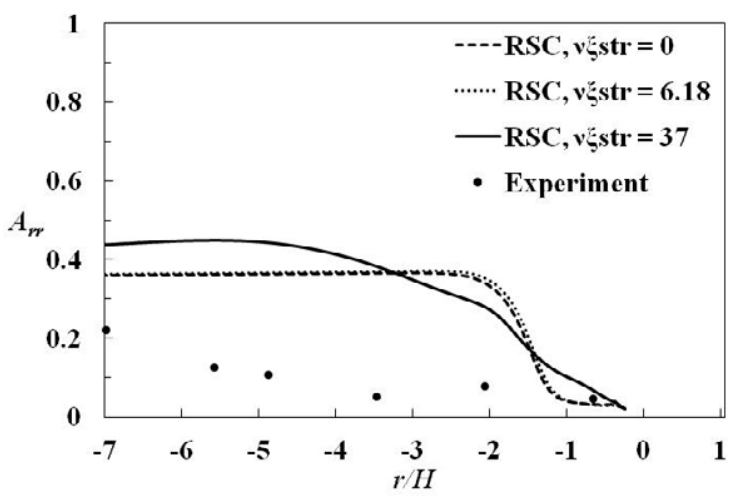

(c)

Figure 12: Comparison of $A_{r r}$ predictions of the RSC model with decoupled and coupled simulations with experimentally measured values at (a) $z_{s} / H=0.75$, (b) $z_{s} / H=0.42$, and (c) $z_{s} / H=0.08$. 


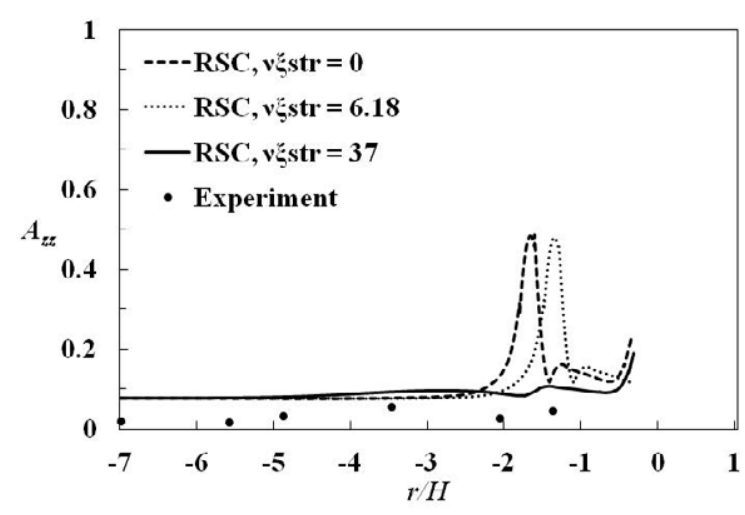

(a)

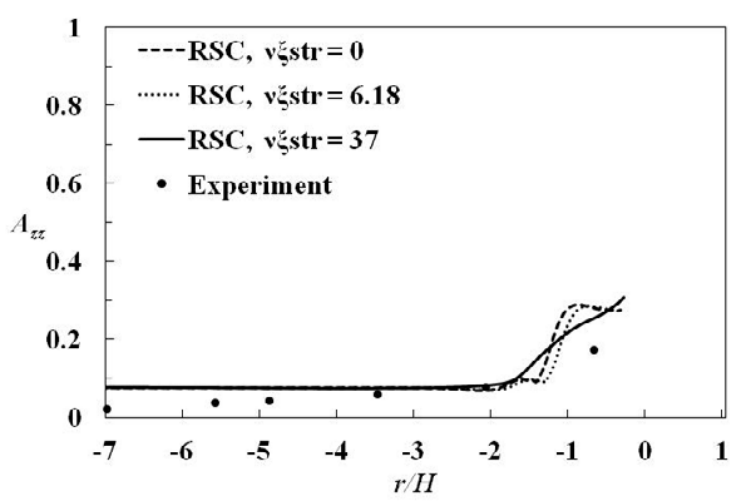

(c)

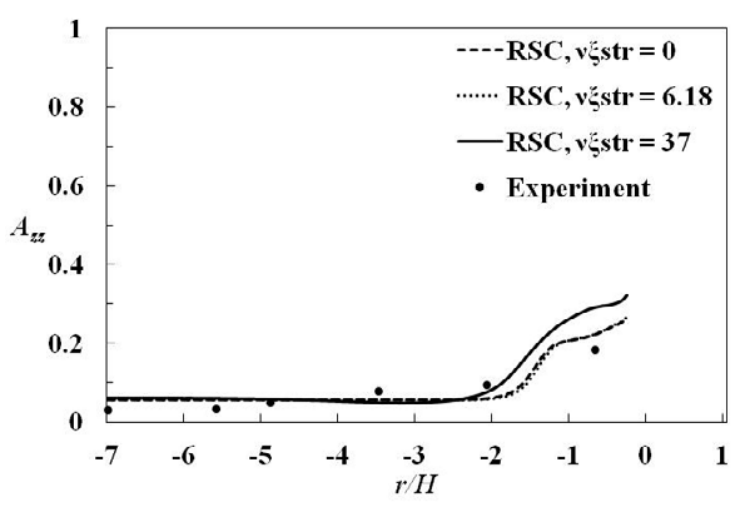

(e)

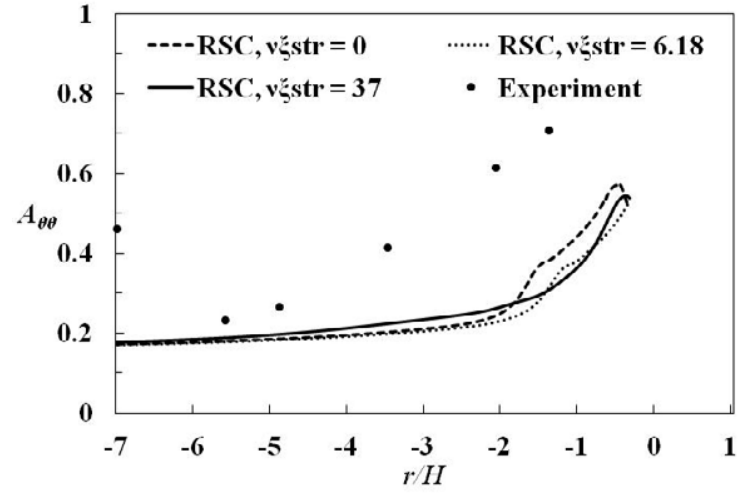

(b)

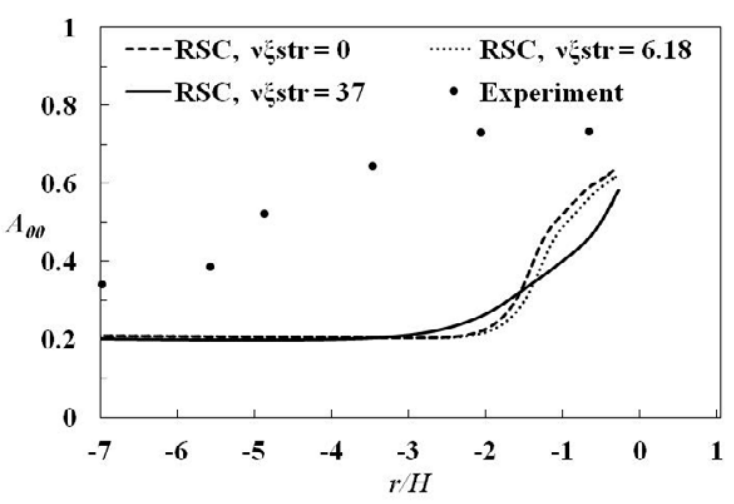

(d)

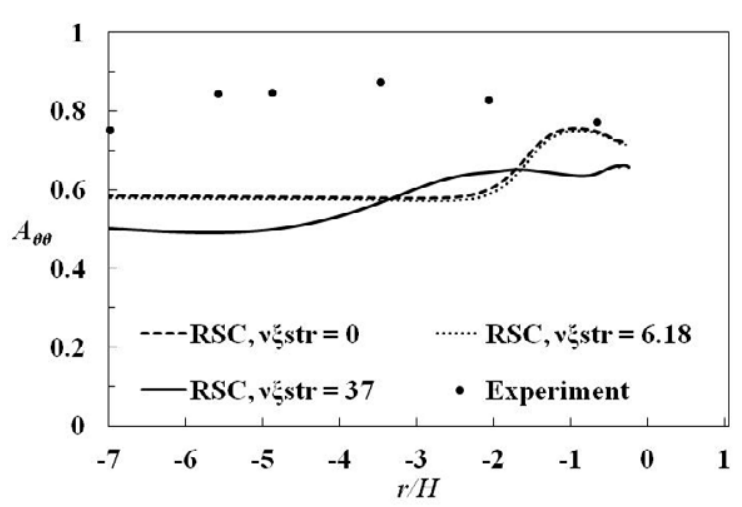

(f)

Figure 13: Comparison of $A_{z z}$ and $A_{\theta \theta}$ predictions of the RSC model using decoupled and coupled simulations with experimentally measured values at (a), (b) $z_{s} / H=0.75$, (c), (d) $z_{s} / H=0.42$, and (e), (f) $z_{s} / H=0.08$. 


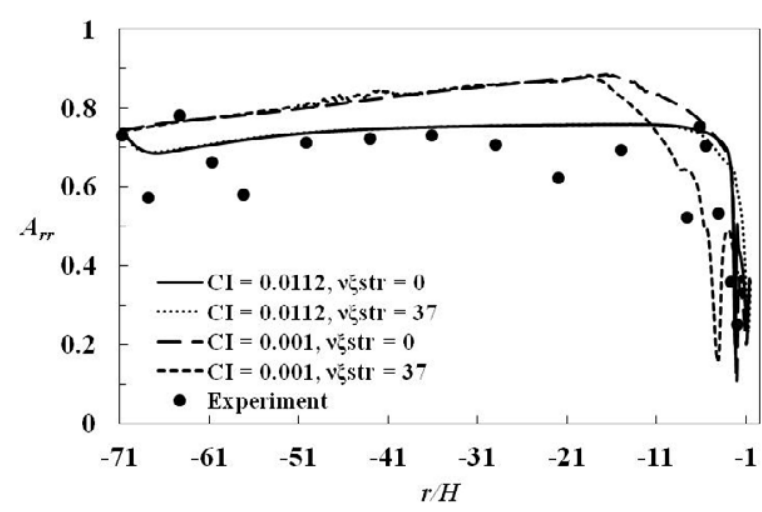

(a)

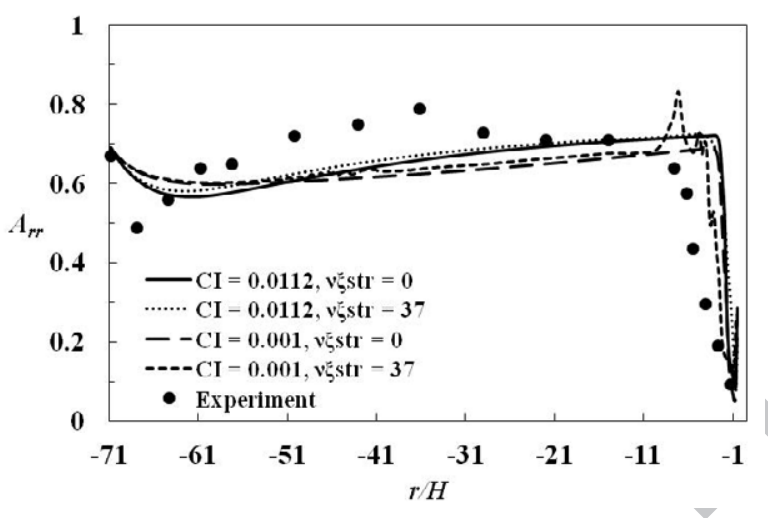

(c)

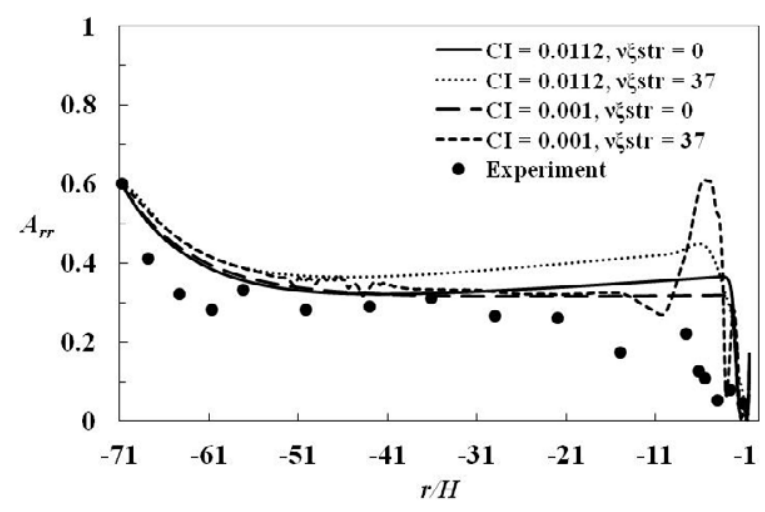

(e)

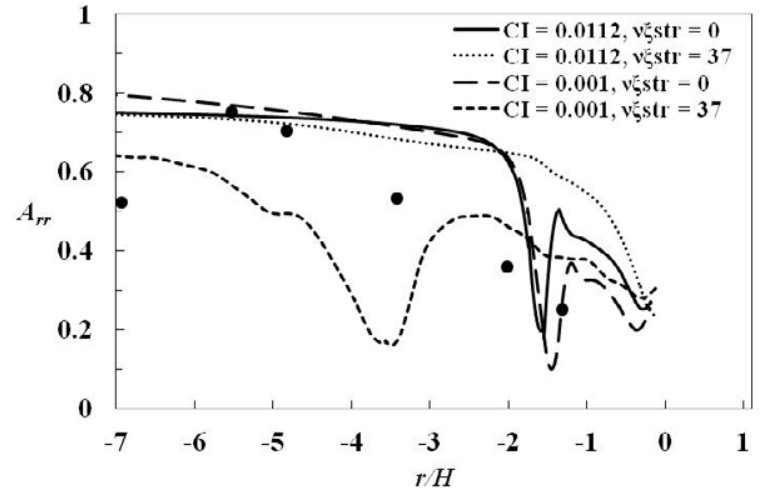

(b)

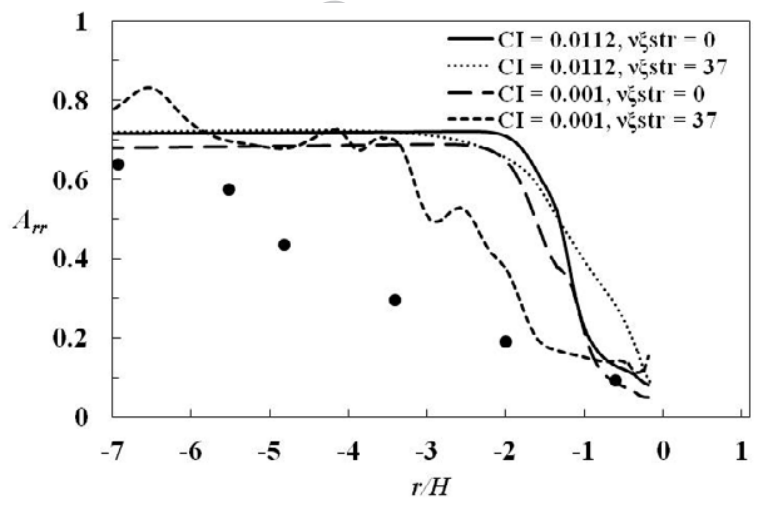

(d)

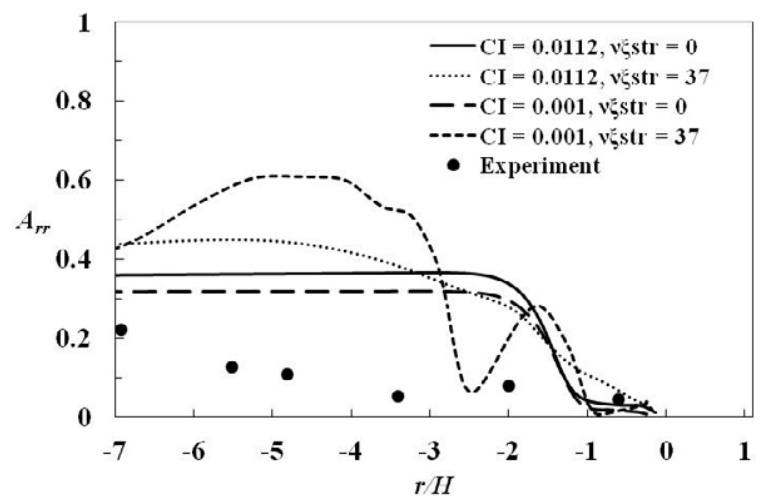

(f)

Figure 14: $A_{r r}$ predictions of coupled and decoupled RSC model using $C_{I}=0.0112$ and $C_{I}=0.001$ at (a), (b) $z_{s} / H=0.75$, (c), (d) $z_{s} / H=0.42$, and (e), (f) $z_{s} / H=0.08$. 


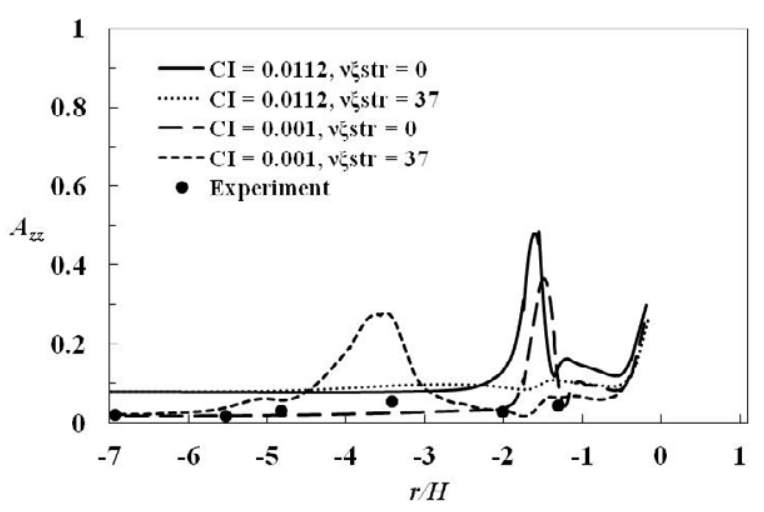

(a)

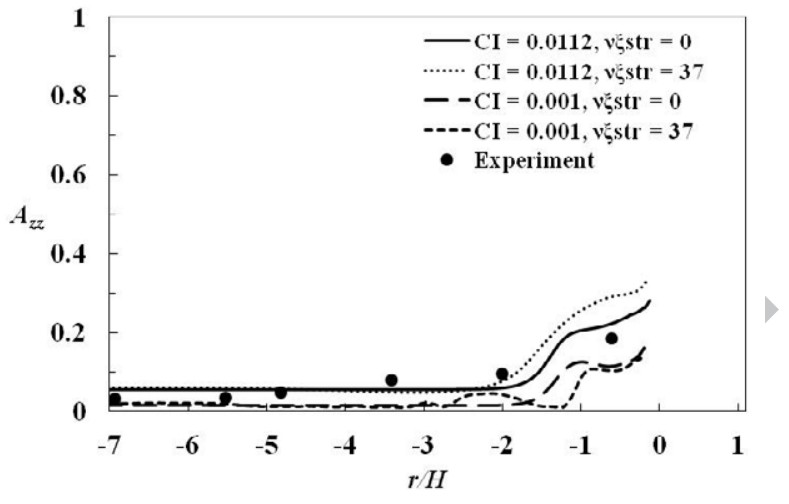

(b)

Figure 15: $A_{z z}$ predictions of coupled and decoupled RSC model using $C_{I}=0.0112$ and $C_{I}=0.001$ at (a) $z_{s} / H=0.75$ and (b) $z_{s} / H=0.08$. 